Electronic version of an article published in

International Journal of Algebra and Computation

Vol. 18, No. 8 (2008) 1283-1319

() World Scientific Publishing Company

Article DOI: 10.1142/S0218196708004913

Journal URL: http://www.worldscinet.com/ijac/ijac.shtml

\title{
Equational complexity of the finite algebra membership problem
}

\author{
George F. McNulty \\ Department of Mathematics, University of South Carolina \\ Columbia SC 29208 USA \\ mcnulty@math.sc.edu \\ Zoltán Székely \\ Division of Mathematical Sciences, College of Natural and Applied Sciences \\ University of Guam UOG Station, Mangilao, GU 96923, USA \\ zszekely@uguam.uog.edu \\ Ross Willard* \\ Department of Pure Mathematics, University of Waterloo \\ Waterloo, Ontario N2L 3G1, Canada \\ rdwillar@uwaterloo.ca
}

Received 28 July 2006

Revised 5 August 2008

Communicated by J. Meakin

In Celebration of the Accomplishments of Béla Csákány

\begin{abstract}
We associate to each variety of algebras of finite signature a function on the positive integers called the equational complexity of the variety. This function is a measure of how much of the equational theory of a variety must be tested to determine whether a finite algebra belongs to the variety. We provide general methods for giving upper and lower bounds on the growth of equational complexity functions and provide examples using algebras created from graphs and from finite automata. We also show that finite algebras which are inherently nonfinitely based via the shift automorphism method cannot be used to settle an old problem of Eilenberg and Schützenberger.
\end{abstract}

Keywords: Equational complexity, variety of algebras, inherently nonfinitely based, shift automorphism method, graph algebra, automatic algebra

2000 Mathematics Subject Classification. 08B05, 03C05, 68Q17

\section{Introduction}

An algebra is a nonempty set equipped with a system of finitary operations. Each algebra has a signature to index its system of fundamental operations. Such a

*The third author was supported by an NSERC grant 
signature is a set of operation symbols, each with given finite rank.

A variety is a class of algebras, all of the same signature, which is closed with respect to the formation of homomorphic images, subalgebras, and direct products of arbitrary systems of algebras. According to a classical theorem of Garrett Birkhoff [6], varieties are exactly those classes of algebras which can be axiomatized by sets of equations. Thus, the classification of algebras into varieties is natural from both the algebraic point of view and from the point of view of mathematical logic.

A basic task arising from this classification scheme is to determine whether an algebra $\mathbf{B}$ belongs to a variety $\mathcal{V}$. For the moment regard $\mathcal{V}$ as fixed.

The Finite Algebra Membership Problem for $\mathcal{V}$

Find a method for determining of a given finite algebra $\mathbf{B}$ whether $\mathbf{B} \in \mathcal{V}$.

In case the signature of $\mathcal{V}$ is finite, this problem can be construed as a computational problem since, at least up to isomorphism, finite algebras of finite signature are suitable as inputs for algorithms. In this paper our primary concern will be with locally finite varieties of finite signature and, in particular, with varieties generated by a single finite algebra of finite signature.

The Finite Algebra Membership Problem has a number of interesting variations. For example, fix a (finite) signature.

\section{The Finite Algebra Membership Problem: Two Algebra VARIANT}

Find a method for determining of two given finite algebras $\mathbf{A}$ and $\mathbf{B}$ whether $\mathbf{B}$ belongs to the variety $\mathcal{V}$ generated by $\mathbf{A}$.

The classical work of Birkhoff $[6,7]$ offers three avenues to approach the Finite Algebra Membership Problem:

Avenue A. Determine whether $\mathbf{B}$ is a homomorphic image of some appropriate free algebra of $\mathcal{V}$.

Avenue B. Determine whether $\mathbf{B}$ satisfies the equations true in $\mathcal{V}$.

Avenue C. Characterize the finite subdirectly irreducible algebras in $\mathcal{V}$ and determine whether $\mathbf{B}$ is a subdirect product of such algebras.

As early as 1952, Jan Kalicki [15] observed, by way of Avenue A, that there is a brute force algorithm for solving the Two Algebra Variant of the Finite Algebra Membership Problem - and hence the Finite Algebra Membership Problem for each finitely generated variety $\mathcal{V}$-at least for finite signatures. In 2000 Cliff Bergman and Giora Slutzki [5] organized this brute force method intelligently enough so that its time-complexity is no worse than on the order of $2^{2^{p(n)}}$, where $n$ is the size of the input algebra $\mathbf{B}$ and $p(n)$ is some polynomial depending on the variety $\mathcal{V}$. Avenue $\mathrm{C}$ lies at the heart of the recent constructions of Székely [36,37], of Jackson and McKenzie [14], and of Kozik [20] of finitely generated varieties whose Finite Algebra Membership Problems have high computational complexity. Kozik 
has recently announced the construction of a finitely generated variety whose finite algebra membership problem is doubly exponentially complete.

In this paper we focus on the equational perspective of Avenue B. Let $\mathcal{V}$ be a variety of finite signature and let $\mathbf{B}$ be a finite algebra. To determine whether $\mathbf{B} \in \mathcal{V}$ it is not necessary to consider all the equations true in $\mathcal{V}$. Up to isomorphism there are only finitely many algebras of the same cardinality as B. For each such algebra that fails to belong to $\mathcal{V}$ pick an equation true in $\mathcal{V}$ which fails in the algebra. In this way, a finite set of equations has been assembled. If they are all true in $\mathbf{B}$, then $\mathbf{B} \in \mathcal{V}$ and otherwise $\mathbf{B} \notin \mathcal{V}$. This insight leads to the definition of the equational complexity of a variety.

The length of an equation is the total number of occurrences of operation symbols (including constant symbols) and variables in the equation. So the associative law $x \cdot(y \cdot z) \approx(x \cdot y) \cdot z$ has length 10 . The length of an equation is just its length as a string of symbols were it rendered as the concatenation of two terms each expressed in prefix (Polish) notation. [In this notation the familiar associative law becomes $\cdot x \cdot y z \cdot x y z$.]

The equational complexity of a variety $\mathcal{V}$ of finite signature is the function $\beta_{\mathcal{V}}$ from the positive integers into the natural numbers so that $\beta_{\mathcal{V}}(n)$ is the smallest natural number $\ell$ such that any algebra $\mathbf{B}$ of cardinality less than $n$ belongs to $\mathcal{V}$ if and only if each equation of length less than $\ell$ which is true in $\mathcal{V}$ is also true in $\mathbf{B}$. Evidently, every variety of finite signature has an equational complexity function. For an algebra $\mathbf{A}$ we use $\beta_{\mathbf{A}}$ to denote $\beta_{\mathcal{V}}$ where $\mathcal{V}$ is the variety generated by $\mathbf{A}$.

In defining $\beta_{\mathcal{V}}$ we have used length as a measure of complexity of equations. Other measures can also be used (for example, the depth of an equation) to arrive at other measures of the equational complexity of a variety. Although such choices change the details, the main thrust of our findings would be much the same.

The equational complexity of a variety is related to the computational complexity of its Finite Algebra Membership Problem. Let $\mathcal{V}$ be a finitely generated variety. In the final section of this paper we will prove that

$$
n^{\beta_{\mathcal{V}}(n+1)+n}
$$

dominates the time complexity of the Finite Algebra Membership Problem of $\mathcal{V}$.

From this point, we stipulate that all signatures are finite.

For a variety $\mathcal{V}$ we denote the class of finite members of $\mathcal{V}$ by $\mathcal{V}_{\text {fin }}$. The equational complexity function $\beta \mathcal{V}$ depends only on $\mathcal{V}_{\text {fin }}$. The class $\mathcal{V}_{\text {fin }}$ is a pseudovariety in the sense that it is a class of finite algebras closed with respect to the formation of homomorphic images, subalgebras, and direct products of arbitrary finite systems of algebras. We recall, see Reiterman [31], that not all pseudovarieties arise as the class of finite members of some variety.

A variety $\mathcal{V}$ is finitely based provided there is a finite set $\Gamma$ of equations so that $\mathcal{V}$ is the class of all algebras in which each equation in $\Gamma$ is true. An algebra is finitely based if and only if the variety it generates is finitely based. Evidently, $\beta \mathcal{V}$ is dominated by some constant function if $\mathcal{V}$ is finitely based. Consider the converse. 
Suppose that $m$ is a natural number such that $\beta_{\mathcal{V}}(n) \leq m$ for all $n$. Up to renaming variables, the set $\Gamma$ of equations true in $\mathcal{V}$ with length no more than $m$ is a finite set. Let $\mathcal{W}$ be the variety axiomatized by $\Gamma$. Then $\mathcal{W}$ is a finitely based variety, $\mathcal{V}_{\text {fin }}=\mathcal{W}_{\text {fin }}$, and, as a consequence, $\beta_{\mathcal{V}}=\beta_{\mathcal{W}}$. However, we see no way to draw the conclusion that $\mathcal{V}$ is itself finitely based. Even in the case when $\mathcal{V}$ is locally finite (so that $\mathcal{V}$ is generated by $\mathcal{V}_{\text {fin }}$ ), we are unable to conclude that $\mathcal{W}$ is locally finite.

In their investigations of pseudovarieties, Eilenberg and Schützenberger [10] posed the following problem (which we cast here in the language of equational complexity):

The Problem of Eilenberg and Schützenberger (1976)

Let $\mathbf{A}$ be a finite algebra of finite signature. Must $\mathbf{A}$ be finitely based if $\beta_{\mathbf{A}}$ is dominated by a constant?

A variety $\mathcal{V}$ is said to be inherently nonfinitely based provided $\mathcal{V}$ is locally finite but included in no finitely based locally finite variety. An algebra is inherently nonfinitely based if and only if it belongs to some locally finite variety but to no locally finite finitely based variety. Robert Cacioppo [8] has pointed out that if $\mathbf{A}$ is a finite algebra and $\beta_{\mathbf{A}}$ is dominated by a constant function, then either $\mathbf{A}$ is finitely based or else it is inherently nonfinitely based. So the Problem of Eilenberg and Schützenberger can be rephrased as

\section{The Problem of Eilenberg and Schützenberger}

Is there an inherently nonfinitely based finite algebra of finite signature whose equational complexity is dominated by a constant function?

The same problem can be asked for locally finite varieties instead of finite algebras.

In 1987 Mark Sapir [32-34] characterized the inherently nonfinitely based finite semigroups. It follows from his work that none of them have equational complexities dominated by a constant. Almost all the other examples of inherently nonfinitely based finite algebras in the literature fulfill the conditions of the shift automorphism method of Baker, McNulty, and Werner [2]. We prove in the next section that no such algebras have equational complexities dominated by a constant. The principal examples of an inherently nonfinitely based finite algebra whose equational complexity might be dominated by a constant are the finite nonassociative bilinear algebras over finite fields constructed by Isaev [13].

The remainder of this paper supplies some general conditions that compel equational complexity functions to grow and others which bound the rapidity of this growth. Since most finite algebras, including groups, rings, lattices, vector spaces, Boolean algebras, ..., encountered in classical algebra are finitely based, we see that finite algebras exhibiting equational complexity growing too fast to be dominated by a constant must be found further afield.

We apply our results to algebras arising from graphs and from finite automata. Let $\mathbf{G}=\langle V, E\rangle$ be a graph, with loops at the vertices allowed. The graph 
algebra $\mathbf{A}_{\mathbf{G}}$ is the algebra with universe $A_{G}=V \cup\{0\}$, where we stipulate that $0 \notin V$, and whose only operation $\cdot$ is binary and defined by

$$
u \cdot v= \begin{cases}u & \text { if there is an edge of } \mathbf{G} \text { from } u \text { to } v \\ 0 & \text { otherwise }\end{cases}
$$

Observe that this definition is meaningful for directed graphs as well. Graph algebras were introduced by Caroline Shallon in her dissertation [35], see also [18, 28]. Perhaps the graph algebra to make the earliest appearance is the 3-element algebra $\mathbf{A}_{\mathbf{M}}$ associated with the graph $\mathbf{M}$ displayed in Figure 1.

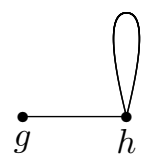

M

Fig. 1. Murskiǐ's Graph M

Murskiı̌ [29] proved that $\mathbf{A}_{\mathbf{M}}$ is nonfinitely based and later [30] that it is inherently nonfinitely based. The finitely based finite graph algebras were given a forbidden subgraph characterization by Baker, McNulty, and Werner [3] and all those which are not finitely based were shown to be inherently nonfinitely based. Similar characterizations have been found by Dejan Delić [9] for flat graph algebras and Brian Walter $[39,40]$ for algebras of directed graphs with loops at all vertices.

A finite automaton is usually described as a five-tuple $\langle\Sigma, Q, \delta, q, F\rangle$, where $\Sigma$ and $Q$ are disjoint finite sets called, respectively, the alphabet and the set of states, $q \in Q$ is called the initial state, $F \subseteq Q$ is the set of final states, and $\delta: \Sigma \times Q \rightarrow Q$ is called the transition function. The designation of initial and final states will play no role here and we relax the constraint on $\delta$ allowing its domain to be a subset of $\Sigma \times Q$. It would be reasonable to call such an object a partial automaton. We make a partial automaton into an automatic algebra by setting the universe to be $\Sigma \cup Q \cup\{0\}$, where we stipulate that $0 \notin \Sigma \cup Q$, and letting the only operation $\cdot$, which is binary, be defined so that

$$
a \cdot r= \begin{cases}s & \text { if } \delta(a, r)=s \\ 0 & \text { otherwise }\end{cases}
$$

Automata are usually depicted as directed graphs whose vertices are the states and whose edges are labeled with the letters from the alphabet $\Sigma$ in a manner to reflect the transition function $\delta$. Perhaps the 7-element automatic algebra $\mathbf{L}$ of Roger Lyndon was the earliest to appear. It is displayed in Figure 2.

In 1954 Lyndon [23] showed that $\mathbf{L}$ is not finitely based. This was the earliest example of a nonfinitely based finite algebra. This algebra fails to be inherently 


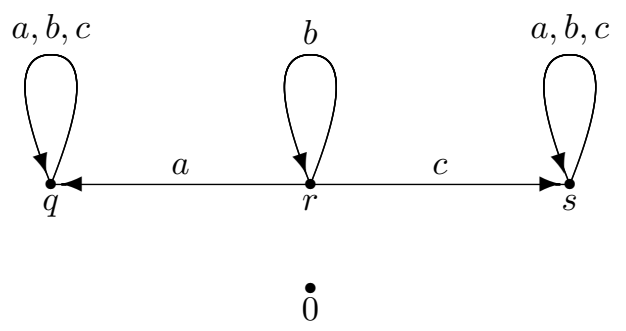

Fig. 2. Lyndon's Automatic Algebra L

nonfinitely based, as demonstrated by Bajusz, McNulty, and Szendrei [1]. Automatic algebras also arose in McKenzie's solution to Tarski's Finite Basis Problem [25-27] and they were considered in 1994 by Keith Kearnes and Ross Willard in [17, Section 3], where they were shown to be 2-step strongly solvable.

We investigate the equational complexity of graph algebras and of automatic algebras. For nonfinitely based finite graph algebras we are able to show that the equational complexity dominates a strictly increasing linear function and is dominated by a quadratic function. For automatic algebras our results are limited to seven examples, one being Lyndon's automatic algebra. For six these examples we provide a linear upper bound on the equational complexity (the remaining bound is quadratic) and, except in the case of Lyndon's algebra, a strictly increasing linear lower bound as well.

An element 0 of an algebra $\mathbf{A}$ is said to be absorbing provided for all operation symbols $Q$ and all $a_{0}, \ldots, a_{r-1} \in A$, where $r$ is the rank of $Q$, we have $Q^{\mathbf{A}}\left(a_{0}, \ldots, a_{r-1}\right)=0$ whenever $0 \in\left\{a_{0}, \ldots, a_{r-1}\right\}$. Graph algebras and automatic algebras have absorbing elements. It is clear that an algebra can have at most one absorbing element, provided the algebra has a basic operation of rank at least 2 .

Finite algebras whose equational complexity exhibits very rapid growth have recently been found by Gábor Kun and Vera Vértesi [21] and by Marcin Kozik [20]. In their work, Kun and Vértesi as well as Kozik consider flat algebras-these are algebras supplied with an absorbing element 0 and, among other operations, a meet operation which imposes on the algebra the structure of a height 1 semilattice with least element 0 . Kun and Vértesi consider flat hypergraph algebras (constructed from hypergraphs in manner extending how Shallon constructed algebras from graphs) while Kozik considers flat algebras associated with Turing machines (a variation of McKenzie's construction [26]). Kun and Vértesi construct examples for arbitrarily large $d$ of finite algebras whose equational complexity is roughly a polynomial of degree $d$. Kozik is able to exhibit a finite algebra with at least exponential equational complexity.

Much of the work presented here grew out of Székely's dissertation [36]. A key new contribution in this paper is Theorem 1 and its proof, found in the next section. The construction in the proof of that theorem has replaced Székely's "Moebius Torus 
algebra" construction enabling us to obtain lower bounds on equational complexity that are more widely applicable.

We are in debt to the referee for a very detailed reading of our paper and for plentiful and cogent suggestions to improve the exposition of this paper.

\section{The shift automorphism method and varieties inherently nonfinitely based in the finite sense}

For any variety $\mathcal{V}$ and any natural number $n$, we use $\mathcal{V}^{(n)}$ to denote the variety based on all the equations true in $\mathcal{V}$ in which no more than $n$ distinct variables occur. It is easy to see that $\mathbf{B} \in \mathcal{V}^{(n)}$ if and only if every subalgebra of $\mathbf{B}$ generated by $n$ or fewer elements belongs to $\mathcal{V}$. Birkhoff [6] proved that if $\mathcal{V}$ is a locally finite variety of finite signature and $n$ is any natural number, then $\mathcal{V}^{(n)}$ is finitely based. This entails, for any locally finite variety $\mathcal{V}$ of finite signature, that $\mathcal{V}$ is inherently nonfinitely based if and only if $\mathcal{V}^{(n)}$ is not locally finite for any natural number $n$.

To demonstrate that a variety $\mathcal{W}$ is not locally finite it is enough to find in $\mathcal{W}$ an infinite algebra which is finitely generated. But it may also be possible to give a natural number $p$ and to find in $\mathcal{W}$ arbitrarily large finite $p$-generated algebras. If $\mathcal{W}$ has the latter property we say it fails to be locally finite in the finite sense.

A locally finite variety $\mathcal{V}$ of finite signature is inherently nonfinitely based in the finite sense if and only if $\mathcal{V}^{(n)}$ fails to be locally finite in the finite sense for every natural number $n$.

From Sapir's work it follows that every inherently nonfinitely based finite semigroup is inherently nonfinitely based in the finite sense. By Theorem 1 below, the same applies to those finite algebras shown to be inherently nonfinitely based with the help of the shift automorphism method of Baker, McNulty, and Werner.

Freese, McNulty, and Nation [11,12] have adapted the shift automorphism method to lattices and used the adaptations to provide examples of inherently nonfinitely based lattice varieties. It is not clear whether these are also inherently nonfinitely based in the finite sense.

Finally, I. M. Isaev [13] has given examples of inherently nonfinitely based finite nonassociative rings and bilinear algebras over finite fields. We do not know whether these must also be inherently nonfinitely based in the finite sense.

\section{Open Problem}

Is there a finite algebra which is inherently nonfinitely based, but which fails to be inherently nonfinitely based in the finite sense?

Let $\mathbf{A}$ be an algebra with an absorbing element 0 . We refer to an element of $A$ as proper if it is not 0 . We say that an $n$-tuple $\left(a_{0}, \ldots, a_{n-1}\right)$ of elements of $A$ is proper if $a_{i}$ is proper for all $i<n$. Regarding an $n$-ary operation $F$ on $A$ as a set of $n+1$-tuples, we take the proper part of $F$ to be the set of all proper tuples belonging to $F$.

Theorem 1. Let $\mathbf{A}$ be an infinite locally finite algebra with only finitely many 
fundamental operations, with an absorbing element 0 , and with an automorphism $\sigma$ such that

a. $\{0\}$ is the only $\sigma$-orbit of $\mathbf{A}$ that is finite;

b. the proper part of $F$ is partitioned by $\sigma$ into only finitely many orbits, for each fundamental operation $F$ of $\mathbf{A}$;

c. $f(a)=\sigma(a)$ for some proper element $a$ of $A$ and some nonconstant unary polynomial function $f$ of $\mathbf{A}$.

Then $\mathbf{A}$ is inherently nonfinitely based in the finite sense.

Proof. Let $\mathcal{V}$ denote the variety generated by $\mathbf{A}$.

Let $n$ be any natural number larger than the rank of any fundamental operation of $\mathbf{A}$. We will find a natural number $p$ and finite algebras $\mathbf{B}_{n, k}$ for infinitely many $k$ such that each $\mathbf{B}_{n, k} \in \mathcal{V}^{(n)}$, each $\mathbf{B}_{n, k}$ has at least $k$ elements, and each $\mathbf{B}_{n, k}$ is generated by a set of $p$ elements. We will also prove that $\mathcal{V}$ is locally finite.

Let $a$ and $b$ be proper elements of $A$. We say that $a$ and $b$ are operationally related provided they are entries in a tuple of proper elements that belongs to some fundamental operation of $\mathbf{A}$. We also say that $a$ is an essential element of $A$ if it belongs to such a tuple. Let $E$ denote the set of essential elements of $\mathbf{A}$. The set $E$ is $\sigma$-invariant and so it is partitioned by $\sigma$ into orbits. Since $\mathbf{A}$ has only finitely many fundamental operations, in view of condition (b) the set $E$ is partitioned into only finitely many $\sigma$-orbits. Let $m$ be the number of $\sigma$-orbits into which $E$ is partitioned. Pick representatives $a_{0}, a_{1}, \ldots, a_{m-1}$ from these orbits so that $a_{0}=a$ where $a$ is the element mentioned in condition (c). Arrange the elements of $E$ in a sequence

$$
\ldots, a_{-2}, a_{-1}, a_{0}, a_{1}, \ldots, a_{m-1}, a_{m}, \ldots
$$

with the order type of the integers so that $\sigma\left(a_{j}\right)=a_{j+m}$. This ordering of $E$ gives meaning to phrases like "to the left of", "to the right of", and "the distance between" when referring to elements of $E$. This ordering of $E$ is preserved under $\sigma$.

According the condition (b) there are up to translations by $\sigma$ only finitely many pairs of operationally related elements. Let $d$ be the maximum distance between any two operationally related elements of $E$. We insist that the choice of representatives of the $\sigma$-orbits and their ordering has been made so that the resulting parameter $d$ is as small as possible. The numbers $m$ and $d$ depend only on $\mathbf{A}$ and $\sigma$.

A third parameter plays a role in our proof. Consider any element $a_{i} \in E$. Let $X=\left\{a_{j} \mid i \leq j\right\}$. The subalgebra of $\mathbf{A}$ generated by $X$ may include elements of $E$ to the left of $a_{i}$. Any such elements must, however, be generated by $\left\{a_{j} \mid i \leq j<i+d\right\}$. This set is finite. Since $\mathbf{A}$ is locally finite, we conclude that $X$ can only generate finitely many elements to the left of $a_{i}$. Let $w_{i}$ denote the distance between $a_{i}$ and the element furthest to the left which is generated by $X$. Considering that $\sigma$ is an automorphism of $\mathbf{A}$, we see that $w_{0}, w_{1}, \ldots, w_{m-1}$ are the only numbers arising in this way. Let $w_{L}$ be the largest of these numbers. Performing the same analysis on 
the right instead of the left will produce another $m$-sequence of numbers. Let $w_{R}$ be the largest number appearing in this sequence

Let $\tau$ be an automorphism of $\mathbf{A}$. We say a subalgebra $\mathbf{S}$ of $\mathbf{A}$ is $\tau$-decomposable if there is a subalgebra $\mathbf{S}_{0}$, called a core of the decomposition, such that

$$
S=\bigcup_{i \in \mathbb{Z}} \tau^{i}\left(S_{0}\right)
$$

and no element of $S_{0}$ is operationally related to any element in any nontrivial $\tau$-translate of $S_{0}$. This means that for any basic operation $F$ and any elements $s_{0}, \ldots, s_{u-1} \in S$ such that $F\left(s_{0}, \ldots, s_{u-1}\right) \neq 0$ we have $\left\{s_{0}, \ldots, s_{u-1}\right\} \subseteq \tau^{i}\left(S_{0}\right)$ for some unique integer $i$. Evidently, every $\tau$-decomposable subalgebra of $\mathbf{A}$ is $\tau$ invariant and it is partitioned into $\tau$-orbits.

Let $\ell$ be the smallest natural number such that $\ell m>n\left(d+w_{L}+w_{R}\right)$. From this point we reserve $\tau$ to denote $\sigma^{\ell}$. Let $m^{\prime}=\ell m$. This is the number of $\tau$-orbits into which $E$ is partitioned.

Claim 2. The union of any $n \tau$-orbits of $E$ generates a $\tau$-decomposable subalgebra of $\mathbf{A}$ whose core is n-generated.

Proof. Suppose that $Y \subseteq E$ is the union of $n \tau$-orbits. Let $y_{0} \in Y$. Scanning the elements of $Y$ to the right we read the sequence

$$
y_{0}, y_{1}, \ldots, y_{n-1}, y_{n}=\tau\left(y_{0}\right)
$$

The distance between $y_{0}$ and $y_{n}$ is $m^{\prime}>n\left(d+w_{L}+w_{R}\right)$. Between adjacent pairs on this sequence there are $n$ gaps. At least one of these gaps must have length at least $d+w_{L}+w_{R}+1$. So $Y$ can be broken up into pieces of size $n$ so that each is the $\tau$ translate of the one to the left and the gaps that separate these pieces are of length at least $d+w_{L}+w_{R}+1$. Let $Y_{0}$ be the piece containing $y_{0}$ and let $\mathbf{S}_{0}$ be the subalgebra generated by $Y_{0}$. Now $S_{0}$ can extend no further than $w_{L}$ points to the left of $Y_{0}$ and no further than $w_{R}$ point to the right. Now put $\mathbf{S}_{j}$ to be the image of $\mathbf{S}_{0}$ under $\tau^{j}$ for each integer $j$. Between any two such translates of $\mathbf{S}_{0}$ there must be a gap of at least $d+1$. It follows that no element of one translate can be operationally related to any element of another translate. Since it is evident that

$$
\bigcup_{j \in \mathbb{Z}} \tau^{j}\left(S_{0}\right)
$$

is the subalgebra generated by $Y$, we see that this subalgebra is $\tau$-decomposable.

Claim 3. $\mathcal{V}$ is locally finite.

Proof. According to [24], it suffices to find a function $b(n)$ on the positive integers so that every $n$-generated subalgebra of $\mathbf{A}$ has no more than $b(n)$ elements. Regarding $n$ as given, let $X$ be any subset of $A$ of cardinality $n$. Apart from perhaps the default element 0 , the only new elements that can be generated from $X$ must be essential 
elements. By insisting that $b(n)>n+1$, we can suppose that $X \subseteq E$. Pick $n \tau$ orbits in such a way that each element of $X$ lies in one of the selected orbits. Thus the subalgebra generated by $X$ is included in the subalgebra generated by these orbits. According to the preceding claim, the latter subalgebra is $\tau$-decomposable. Let $\mathbf{S}_{0}$ be the core of this $\tau$-decomposition. Then

$$
\operatorname{Sg}^{\mathbf{A}} X \subseteq \bigcup_{i \in \mathbb{Z}} \tau^{i} S_{0}
$$

Now $X$ must lie in the union of $n$ translates of $S_{0}$. Since the translates are operationally unrelated they constitute a subalgebra. The means that the size of the subalgebra generated by $X$ can be no bigger than $n$ times the size of $S_{0}$. This number still depends on the cardinality of $S_{0}$, which is itself an $n$-generated subalgebra of $\mathbf{A}$. To bound this number, notice that there are only $m^{\prime} \tau$-orbits. There are $\left(\begin{array}{c}m^{\prime} \\ n\end{array}\right)$ ways to pick $n$ of these orbits. For each such selection we can pick a core algebra for the corresponding $\tau$-decomposable subalgebra. Then $n$ times the size of the largest of these will suffice for $b(n)$.

The set $E \cup\{0\}$ is a subuniverse of $\mathbf{A}$. We consider a partial subalgebra of the $2 k$-fold direct power of this algebra. Let $e$ be any essential element and let $U \subseteq\{0,1, \ldots, 2 k-1\}$. By $e \mid U$ we mean the $2 k$-tuple that has $e$ at the $j^{\text {th }}$ position for all $j \in U$ and 0 at all other positions. Let $\mu$ be the map from the set $\mathbb{Z}$ of integers to $\{0,1, \ldots, 2 k-1\}$ defined by

$$
\mu(\ell) \equiv \ell(\bmod 2 k) \text { for all } \ell \in \mathbb{Z} .
$$

We say that a subset $U \subseteq\{0,1, \ldots, 2 k-1\}$ is contiguous modulo $2 k$ provided it is the image under the map $\mu$ of an interval in $\mathbb{Z}$. Let $G=\{e|U| e \in$ $E$ and $U$ is nonempty contiguous set modulo $2 k$ with no more than $k$ members $\}$. This set is the universe of a partial subalgebra $\mathbf{G}$. We use $\mathbf{G}^{\#}$ to denote the subalgebra of $\mathbf{A}^{2 k}$ obtained by adjoining the constantly 0 tuple to $\mathbf{G}$.

The automorphism $\tau$ of $\mathbf{E}$ extends coordinatewise to an automorphism of $G$. We use $\tau$ to denote this extension as well. $\mathbf{G}$ has another automorphism $\rho$ arising from the cyclic shift of coordinates. We use $\rho$ to denote the cyclic shift on the coordinate set as well. Notice that the collection of $U$ 's that play a role in $G$ is closed with respect to the cyclic shift. Evidently, the two automorphisms $\tau$ and $\rho$ permute: $\tau \circ \rho=\rho \circ \tau$.

Claim 4. $\tau \circ \rho$ partitions the partial algebra $\mathbf{G}$ into $2 k^{2} m^{\prime}$ orbits and these orbits are the congruence classes of some congruence relation of $\mathbf{G}$.

Proof. There are $m^{\prime} \tau$-orbits of $E$ and there are $2 k^{2}$ cyclically contiguous nonempty subsets of $\{0,1, \ldots, 2 k-1\}$ of cardinality no more than $k$. The conclusion that the number of orbits is $2 k^{2} m^{\prime}$ is routine. 
To see that the underlying equivalence relation is a congruence, suppose that $F$ is a fundamental operation symbol and that

$$
\begin{aligned}
F^{\mathbf{G}}\left(b_{0}\left|U_{0}, \ldots, b_{r-1}\right| U_{r-1}\right) & =b_{r} \mid U_{r} \\
F^{\mathbf{G}}\left(d_{0}\left|V_{0}, \ldots, d_{r-1}\right| V_{r-1}\right) & =d_{r} \mid V_{r} \\
(\tau \circ \rho)^{e_{j}} b_{j} \mid U_{j} & =d_{j} \mid V_{j} \text { for } j<r
\end{aligned}
$$

We need to verify that $b_{r} \mid U_{r}$ and $d_{r} \mid V_{r}$ lie in the same orbit.

The definition of $G$ and the fact that 0 is an absorbing element entail

$$
\begin{aligned}
F^{\mathbf{A}}\left(b_{0}, \ldots, b_{r}\right) & =b_{r} \\
F^{\mathbf{A}}\left(d_{0}, \ldots, d_{r}\right) & =d_{r} \\
U_{0} \cap U_{1} \cap \cdots \cap U_{r-1} & =U_{r} \\
V_{0} \cap V_{1} \cap \cdots \cap V_{r-1} & =V_{r} \\
\tau^{e_{j}}\left(b_{j}\right) & =d_{j} \text { for all } j<r \\
\rho^{e_{j}}\left(U_{j}\right) & =V_{j} \text { for all } j<r
\end{aligned}
$$

So we will have the desired conclusion once we show that $e_{0}=e_{1}=\cdots=e_{r-1}$.

Recall that $n$ is greater than the rank of any fundamental operation. So $b_{0}, \ldots, b_{r-1}$ cannot be in more than $n \tau$-orbits. Of course, $d_{0}, \ldots, d_{r-1}$ lie in the same $\tau$-orbits. So all these essential elements belong to a single $\tau$-decomposable subalgebra. Now $b_{0}, \ldots, b_{r-1}$ are operationally related. So they must lie in one of the translates of the core. Likewise, $d_{0}, \ldots, d_{r-1}$ must also lie in one of the translates of the core. Therefore there is an integer $e$ so that $\tau^{e}$ carries the translate containing the $b_{j}$ 's to the translate containing the $d_{j}$ 's. Hence $e=e_{0}=e_{1}=\cdots=e_{r-1}$.

The congruence of $\mathbf{G}$ whose congruence classes are the $\tau \circ \rho$ orbits will be denoted by $\theta$. Let $\mathbf{G}_{n, k}$ be the algebra made by adding a default absorbing element to $\mathbf{G} / \theta$. The cardinality of $\mathbf{G}_{n, k}$ is $2 k^{2} m^{\prime}+1$.

Claim 5. $\mathbf{G}_{n, k} \in \mathcal{V}^{(n)}$.

Proof. Consider $n$ proper elements of $G / \theta$, that is $n \tau \circ \rho$-orbits of G. Corresponding to each of these $\tau \circ \rho$-orbits is a $\tau$-orbit in $E$. In this way we obtain $n \tau$-orbits. The subalgebra $\mathbf{D}$ generated by the union of these orbits is $\tau$-decomposable. Let $\mathbf{S}$ denote the core of this decomposition. Now we can pick elements $b_{0}, \ldots, b_{n-1}$ of $S$ and appropriate subsets $U_{0}, \ldots, U_{n-1}$ of $\{0, \ldots, 2 k-1\}$ so that $b_{0}\left|U_{0}, \ldots, b_{n-1}\right| U_{n-1}$ is a system of representatives of our original $\tau \circ \rho$-orbits. Let $\mathbf{H}$ be the subalgebra of $\mathbf{G}^{\#}$ generated by $\left\{b_{0}\left|U_{0}, \ldots, b_{n-1}\right| U_{n-1}\right\}$. Let $\pi$ be the map from $H$ to $G_{n, k}$ defined so that each proper element of $H$ is assigned the $\tau \circ \rho$-orbit to which it belongs and the absorbing element of $\mathbf{H}$ is assigned the absorbing element of $\mathbf{G}_{n, k}$. To see that $\pi$ is a homomorphism from $\mathbf{H}$ into $\mathbf{G}_{n, k}$ let $F$ be a basic operation symbol and let $d_{0}\left|V_{0}, \ldots, d_{r-1}\right| V_{r-1}$ be proper elements of $H$, where $r$ is the rank of $F$. Consider 
the following equations:

$$
\begin{aligned}
\pi\left(F^{\mathbf{H}}\left(d_{0}\left|V_{0}, \ldots, d_{r-1}\right| V_{r-1}\right)\right) & =\pi\left(F^{\mathbf{A}}\left(d_{0}, \ldots, d_{r-1}\right) \mid V_{0} \cap \cdots \cap V_{r-1}\right) \\
F^{\mathbf{G}_{n, k}}\left(\pi\left(d_{0} \mid V_{0}\right), \ldots, \pi\left(d_{r-1} \mid V_{r-1}\right)\right) & =F^{\mathbf{G}_{n, k}}\left(d_{0}\left|V_{0} / \theta, \ldots, d_{r-1}\right| V_{r-1} / \theta\right)
\end{aligned}
$$

We would like to show that the four sides of the equations above are all equal. The right side of the second equation is the absorbing element, unless representatives $g_{0}\left|W_{0}, \ldots, g_{r-1}\right| W_{r-1}$ of the $\theta$-classes can be found so that $F^{\mathbf{G}}\left(g_{0}\left|W_{0}, \ldots, g_{r-1}\right| W_{r-1}\right)$ is defined in $\mathbf{G}$. In this case, the $\tau$-decomposability of $\mathbf{D}$ entails that $F^{\mathbf{G}}\left(d_{0}\left|V_{0}, \ldots, d_{r-1}\right| V_{r-1}\right)$ is defined in $\mathbf{G}$ as well. It follows that all four sides of the equations displayed above are equal. Therefore $\pi$ is a homomorphism.

Evidently, our $n$ original elements of $G_{n, k}$ belong to the image of $\pi$ and so the subalgebra they generate belongs to $\mathcal{V}$ since $\mathbf{H} \in \mathcal{V}$.

We have yet to determine $p$ and to define $\mathbf{B}_{n, k}$. The algebras $\mathbf{G}_{n, k}$ almost serve, except for the size of generating sets. The algebras we are looking for will be subalgebras of the $\mathbf{G}_{n, k}$.

Condition (c) of the theorem provides a unary polynomial $f$ and an essential element $a$ so that $f(a)=\sigma(a)$. We need such a polynomial that will do the same with $\tau$. Let $t\left(x, y_{1}, \ldots, y_{r}\right)$ be a term in which $x$ occurs and let $c_{1}, \ldots, c_{r}$ be essential elements so that

$$
f(x)=t^{\mathbf{A}}\left(x, c_{1}, \ldots, c_{r}\right) .
$$

Then a polynomial that works with $\tau$ and $a$ is $f^{\prime}(x)$ displayed below.

$$
t^{\mathbf{A}}\left(t^{\mathbf{A}}\left(\cdots\left(t^{\mathbf{A}}\left(x, c_{1}, \ldots, c_{r}\right) \cdots\right), \sigma^{\ell-2}\left(c_{1}\right), \ldots, \sigma^{\ell-2}\left(c_{r}\right)\right), \sigma^{\ell-1}\left(c_{1}\right), \ldots, \sigma^{\ell-1}\left(c_{r}\right)\right)
$$

That is, $f^{\prime}(a)=\tau(a)$.

Let $t^{\prime}\left(x, y_{1}, \ldots, y_{p-1}\right)$ be a term and $c_{1}, \ldots, c_{p-1}$ be essential elements of $\mathbf{A}$ so that $f^{\prime}(x)=t^{\prime \mathbf{A}}\left(x, c_{1}, \ldots, c_{p-1}\right)$. That is the value of $p$ is one more than the number of "coefficients" in $f^{\prime}$. Notice that $p=\ell r+1$ where $r$ is the number of "coefficients" in the original polynomial $f$. Let $Y_{j}=\{0, \ldots, j-1\}$ for each $j<k$. Let $Z_{j}$ be the preimage of $Y_{j}$ under the cyclic shift. That is

$$
Z_{j}=\{2 k-1,0,1, \ldots, j-2\} .
$$

Let $\mathbf{B}_{n, k}$ be the subalgebra of $\mathbf{G}_{n, k}$ generated by the following elements:

$$
a\left|Y_{k} / \theta, c_{1}\right| Y_{k} / \theta, \ldots, c_{p-1} \mid Y_{k} / \theta .
$$

The only thing that remains is to prove that $\mathbf{B}_{n, k}$ has at least $k$ elements. We do this by showing that the following $k$ distinct elements

$$
a\left|Z_{1} / \theta, a\right| Z_{2} / \theta, \ldots, a \mid Z_{k} / \theta
$$

all belong to $\mathbf{B}_{n, k}$. 
Observe

$$
\begin{aligned}
t^{\prime \mathbf{G}}\left(a\left|Y_{k}, c_{1}\right| Y_{k}, \ldots, c_{p-1} \mid Y_{k}\right) & =t^{\prime \mathbf{A}}\left(a, c_{1}, \ldots, c_{p-1}\right) \mid Y_{k} \\
& =\tau(a) \mid Y_{k} \\
& \theta a \mid Z_{k}
\end{aligned}
$$

and

$$
\begin{aligned}
t^{\prime \mathbf{G}}\left(a\left|Z_{k}, c_{1}\right| Y_{k}, \ldots, c_{p-1} \mid Y_{k}\right) & =t^{\prime \mathbf{A}}\left(a, c_{1}, \ldots, c_{p-1}\right) \mid Y_{k-1} \\
& =\tau(a) \mid Y_{k-1} \\
& \theta a \mid Z_{k-1}
\end{aligned}
$$

So $\mathbf{B}_{n, k}$ has at least $k$ elements. This concludes the proof of the theorem.

The proof just given follows closely the lines of the proof given by Baker, McNulty, and Werner in [2] for their Theorem 1.1. Baker, McNulty, and Werner, in effect, considered the algebra $\mathbf{A}^{\mathbb{Z}}$ and exploited the effect of the shift on $\mathbb{Z}$ on final segments of $\mathbf{B}$. We use a corresponding process on the algebras $\mathbf{A}^{\{0,1, \ldots, 2 k-1\}}$ exploiting the effect of the cyclic shift on $\{0,1, \ldots, 2 k-1\}$ on certain contiguous subsets of the $2 k$-cycle.

For the purposes of understanding equational complexity this proof contains useful information not stated in the theorem. The parameters $d, w_{L}, w_{R}$ and $m$ depend only on the algebra $\mathbf{A}$ and its automorphism $\sigma$. The parameters $\ell$ and $p$ depend on $n$ and this dependence is roughly linear. We record here some of this useful information about the algebras $\mathbf{B}_{n, k}$ that are constructed in the proof above:

(i) $\mathbf{B}_{n, k} \in \mathcal{V}^{(n)}$ for all $k$ and all sufficiently large $n$.

(ii) $k \leq\left|B_{n, k}\right| \leq 2 k^{2} \ell m+1$ for all $k$ and all sufficiently large $n$.

(iii) $\mathbf{B}_{n, k}$ is $p$-generated for all $k$ and all sufficiently large $n$.

(iv) $\mathbf{B}_{n, k} \notin \mathcal{V}$ for all sufficiently large $n$ and all $k$ greater than the cardinality of the algebra $\mathcal{V}$-freely generated by $p$ elements.

The last item on this list is rather inconvenient since $p$ depends on $n$ and the free spectrum function of $\mathcal{V}$ might grow quite rapidly.

In a large number of applications of this theorem to varieties generated by a finite algebra $\mathbf{C}$, the algebra $\mathbf{A}$ turns out to be an easily understood quotient of a subalgebra of $\mathbf{C}^{\mathbb{Z}}$ and the automorphism $\sigma$ derives from the right shift on the direct power. In these cases, the parameters $d, w, m, \ell$, and $p$ can usually be determined.

We say that a locally finite variety $\mathcal{V}$ of finite signature is inherently nonfinitely based by reason of the shift automorphism method when $\mathcal{V}$ has an algebra $\mathbf{A}$ with an automorphism $\sigma$, a unary polynomial $f(x)$ and an element $a$ fulfilling the conditions of this theorem (or equivalently, the conditions of Theorem 1.1 of [2]). 


\section{Lower bounds on equational complexity}

To assess how fast the equational complexity of a variety grows, we employ the following system of comparison. Let $f$ and $g$ be functions from the natural numbers into the real numbers. We say that $f$ eventually dominates $g$ when $g(n) \leq f(n)$ for all sufficiently large values of $n$.

Lemma 6. Let $\mathcal{V}$ be a variety of finite signature and let $d$ and $f$ be functions on the positive real numbers which assign integers to integers, with $d$ strictly increasing and continuous and $f$ monotonically increasing. Suppose that for each sufficiently large natural number $n$ there is an algebra $\mathbf{C}_{n}$ with all the following properties

(1) $\left|C_{n}\right| \leq d(n)$,

(2) $\mathbf{C}_{n} \in \mathcal{V}^{(f(n))}$, and

(3) $\mathbf{C}_{n} \notin \mathcal{V}$

Then $\beta_{\mathcal{V}}(m)$ eventually dominates $f\left(d^{-1}(m-1)-1\right)+1$.

Proof. Let $n_{0}$ be a natural number so that conditions (1)-(3) hold for all $n \geq n_{0}$.

Since for any $n \geq n_{0}$ we have $\mathbf{C}_{n} \notin \mathcal{V}$ there will be equations true in $\mathcal{V}$ which fail in $\mathbf{C}_{n}$, but since $\mathbf{C}_{n} \in \mathcal{V}^{(f(n))}$ any such equations must involve at least $f(n)+1$ distinct variables. In consequence, any equation used to exclude $\mathbf{C}_{n}$ from $\mathcal{V}$ must have length at least $f(n)+1$. Hence

$$
f(n)+1<\beta_{\mathcal{V}}(d(n)+1) \text { for all } n \geq n_{0} .
$$

Now let $m \geq d\left(n_{0}\right)+1$. Pick $n \geq n_{0}$ so that

$$
d(n)+1 \leq m \leq d(n+1)+1 .
$$

Since $d$ is continuous and strictly increasing it follows that

$$
\begin{aligned}
d^{-1}(m-1) & \leq n+1 \\
d^{-1}(m-1)-1 & \leq n \\
f\left(d^{-1}(m-1)-1\right) & \leq f(n) \\
f\left(d^{-1}(m-1)-1\right)+1 & \leq f(n)+1 \\
f\left(d^{-1}(m-1)-1\right)+1 & <\beta_{\mathcal{V}}(d(n)+1) \\
f\left(d^{-1}(m-1)-1\right)+1 & <\beta_{\mathcal{V}}(m) .
\end{aligned}
$$

The last inequality follows from $d(n)+1 \leq m$ because $\beta_{\mathcal{V}}$ is monotonically increasing.

In the lemma above the monotonicity and continuity properties of the functions $d(x)$ and $f(x)$ only need to hold for all large enough values of $x$. If further information is available about the signature a sharper bound can be established. Equations which have at least $f(n)+1$ occurrences of variables will be longer than $f(n)+1$ by an amount that depends of the ranks of the operation symbols of the signature. For 
example, if all the operation symbols are binary then the length of the equations will be $2 f(n)$. This would lead to the larger lower bound $2 f\left(d^{-1}(m-1)-1\right)$.

Since the free spectrum function of a finitely generated variety is dominated by a doubly exponential function of the form $2^{2^{c n}}$, application of Lemma 6 yields the next theorem.

Theorem 7. Let $\mathcal{V}$ be a variety generated by a finite algebra with finitely many fundamental operations. If $\mathcal{V}$ is inherently nonfinitely based by reason of the shift automorphism method, then $\beta_{\mathcal{V}}$ eventually dominates a loglog function. In particular, $\beta_{\mathcal{V}}$ cannot be dominated by a constant function.

Proof. We use the algebras constructed in the proof of Theorem 1. Let $\mathbf{C}_{n}=\mathbf{B}_{n, k}$ where $k$ is one larger that the size of the algebra $\mathcal{V}$-freely generated by $p$ elements. According to the remarks following Theorem 1 we can take $d(x)$ to be a function of the form $2^{2^{c x}}$ and $f(x)=x$. Then $d^{-1}(x)=1 / c \log \log x$. Invoking the Lemma 6 finishes the proof.

It is tempting to pursue a similar analysis when $d(x)$ is dominated by a polynomial. This leads to the consideration of varieties of finite signature whose free spectrum functions are dominated by polynomials. However, in 1980 Joel Berman [4] proved that such varieties are finitely based (and therefore have equational complexity dominated by a constant). Moreover, Berman showed that such varieties have no term function depending on more than $k$ variables, where $k$ is degree of the polynomial dominating the free spectrum function. Keith Kearnes and Emil Kiss [16] give deep results characterizing finitely generated varieties with this last property.

Recall that the Problem of Eilenberg and Schützenberger asks whether there is an inherently nonfinitely based finite algebra with equational complexity dominated by a constant function. No such algebra, should they exist, can fulfill the conditions of the shift automorphism method.

In [26] Ralph McKenzie associated to each Turing machine $\mathcal{T}$ an algebra $\mathbf{A}(\mathcal{T})$ of finite signature so that the variety generated by $\mathbf{A}(\mathcal{T})$ has a finite residual bound if and only if $\mathcal{T}$ halts when launched on the empty tape. McKenzie also noted that in the event that $\mathcal{T}$ does not halt, that $\mathbf{A}(\mathcal{T})$ is inherently nonfinitely based by reason of the shift automorphism method. In case the variety generated by $\mathbf{A}(\mathcal{T})$ has a finite residual bound, Willard [41] proved that $\mathbf{A}(\mathcal{T})$ is finitely based. So we obtain the following corollary of Theorem 7 .

Corollary 8. There is no algorithm which, upon input of a finite algebra $\mathbf{A}$ of finite signature, will determine whether $\beta_{\mathbf{A}}$ is dominated by a constant function.

These results, while they constrain $\beta_{\mathcal{V}}$ to grow, do not ensure very rapid growth. The trouble is that we used the free spectrum to exclude $\mathbf{C}_{n}$ from $\mathcal{V}$. An alternative method using test equations is available in the literature of nonfinitely based varieties. This method requires the discovery of a list $\epsilon_{0}, \epsilon_{1}, \epsilon_{2}, \ldots$ of equations (called 
test equations) true in $\mathcal{V}$ such that $\epsilon_{n}$ fails in $\mathbf{C}_{n}$. In this method, we often use essentially the same algebras as in the shift-automorphism method, but with appropriate test equations available, we may be able to show, for given $n$ that an algebra somewhat smaller than the one provided by the shift-automorphism method lies outside $\mathcal{V}$ but in $\mathcal{V}^{(n)}$.

The next example illustrates this method of test equations.

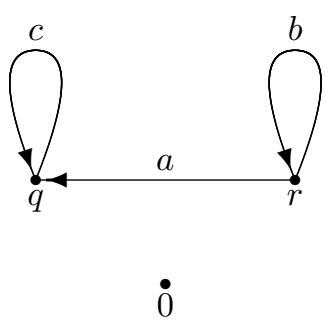

Fig. 3. McKenzie's Automatic Algebra R

The algebra $\mathbf{R}$ illustrated in Figure 3 has six elements: the labels of the vertices ( $q$ and $r$ ), the labels of the edges $(c, a$, and $b$ ), and the default element 0 . The algebra $\mathbf{R}$ has just one operation, which is binary, and that produces by default 0 except in cases like $q=a r$ when a vertex label is produced by combining an edge label with a vertex label, these labels being compatible with Figure 3.

Let $\mathcal{V}$ be the variety generated by $\mathbf{R}$. First we construct an algebra $\mathbf{A} \in \mathcal{V}$ and an automorphism $\sigma$ of $\mathbf{A}$ that fulfills the hypotheses of Theorem 1. Let $\mathbf{a}_{0}$ be the following element of $A^{\mathbb{Z}}$ :

$$
(\ldots, q, q, q, r, r, r, \ldots)
$$

where the rightmost $q$ occurs at coordinate 0 . Let $\mathbf{a}_{1}$ be

$$
(\ldots, c, c, c, a, b, b, b, \ldots)
$$

where the $a$ occurs at coordinate 0 . In general, $\mathbf{a}_{j}$ for any integer $j$ will result from the appropriate shifts of either $\mathbf{a}_{0}$ (in case $j$ is even) or $\mathbf{a}_{1}$ (in case $j$ is odd). So $\mathbf{a}_{-4}$ looks just like $\mathbf{a}_{0}$ except the rightmost $q$ occurs at coordinate $-2=-4 / 2$. Finally, let $A^{\prime}$ consist of all the $\mathbf{a}_{j}$ 's as well as all the $\mathbb{Z}$-tuples in which 0 occurs. Then $A^{\prime}$ is a subuniverse of $\mathbf{R}^{\mathbb{Z}}$. Let $\varphi$ be the equivalence relation on $A^{\prime}$ whose blocks are the singletons $\left\{\mathbf{a}_{j}\right\}$ and the one big block consisting of all $\mathbb{Z}$-tuples in which 0 occurs. Then $\varphi$ is a congruence relation on $\mathbf{A}^{\prime}$. We take $\mathbf{A}=\mathbf{A}^{\prime} / \varphi$. With a minor abuse of notation, we take the elements of $A$ to be the $\mathbf{a}_{j}$ 's and the absorbing element 0 . The automorphism $\sigma$ is essentially the shift: $\sigma\left(\mathbf{a}_{j}\right)=\mathbf{a}_{j+2}$ and $\sigma(0)=0$. The 
parameters that arose in the proof of Theorem 1 have the values:

$$
\begin{aligned}
d & =2 \\
m & =2 \\
w_{L} & =0 \\
w_{R} & =1
\end{aligned}
$$

Furthermore we take $k=3$ and put $\ell=2 n$. With these choices, the algebras $\mathbf{G}_{n, 3}$ constructed in the proof of Theorem 1 will have cardinality $72 n+1$ and they will belong to $\mathcal{V}^{(n)}$. To ensure that $\mathbf{G}_{n, 3} \notin \mathcal{V}$ we present an equation $\epsilon_{n}$ which is true in $\mathbf{R}$ and fails in $\mathbf{G}_{n, 3}$. Here it is:

$$
x_{0} x_{\ell-1} \ldots x_{1} x_{0} x_{\ell-1} \ldots x_{1} x_{0} y \approx x_{0} x_{\ell-1} \ldots x_{1} x_{0} y . \quad\left(\epsilon_{n}\right)
$$

where the association is to the right.

We omit the proof that $\epsilon_{n}$ holds in $\mathbf{R}$. To see that it fails in $\mathbf{G}_{n, 3}$ we put $U=$ $\{0,1,2\}, V=\{1,2,3\}$, and $W=\{2,3,4\}$ and we make the following assignments

$$
\begin{aligned}
\mathbf{a}_{0} \mid U / \theta & \mapsto y \\
\mathbf{a}_{1} \mid U / \theta & \mapsto x_{0} \\
\mathbf{a}_{3} \mid U / \theta & \mapsto x_{1} \\
\vdots & \\
\mathbf{a}_{2 \ell-1} \mid U / \theta & \mapsto x_{\ell-1}
\end{aligned}
$$

Now notice that

$$
\begin{aligned}
\mathbf{a}_{1} \mid U & \equiv \mathbf{a}_{2 \ell+1} \mid V \quad \bmod \theta \\
\mathbf{a}_{3} \mid U & \equiv \mathbf{a}_{2 \ell+3} \mid V \quad \bmod \theta \\
& \vdots \\
\mathbf{a}_{2 \ell-1} \mid U & \equiv \mathbf{a}_{4 \ell-1} \mid V \quad \bmod \theta \\
\mathbf{a}_{1} \mid U & \equiv \mathbf{a}_{4 \ell+1} \mid W \quad \bmod \theta
\end{aligned}
$$

Using these congruences to help in the computation, we see that under this assignment the right side of $\epsilon_{n}$ evaluates to $\mathbf{a}_{2 \ell+2} \mid\{1,2\} / \theta$ while the left side of the equation evaluates to $\mathbf{a}_{4 \ell+2} \mid\{2\} / \theta$. These are distinct, so $\epsilon_{n}$ fails in $\mathbf{G}_{n, 3}$.

By Lemma $6, \beta_{\mathbf{R}}$ eventually dominates the strictly increasing linear function

$$
\frac{1}{72} m-\frac{1}{36}
$$

Since we are only dealing with a binary operation symbol we can do better. We know any equation which has at least $n+1$ occurrences of variables must have 
length at least $2 n$. It follows by the proof of Lemma 6 that $\beta_{\mathbf{R}}(m)$ dominates a strictly increasing linear function

$$
\frac{1}{36} m-\frac{37}{18}
$$

that grows twice as fast. But the essential conclusion that $\beta_{\mathbf{R}}$ dominates a strictly increasing linear function remains the same.

The method used in this example works in many other settings. Notice that the polynomial function stipulated in condition (c) of Theorem 1 played a different role here. Roughly speaking, it was used to construct a violation of one of the infinite list $\epsilon_{0}, \epsilon_{1}, \ldots$ of test equations. Some adjustment seems needed to apply the method of test equations to nonfinitely based finite algebras like Lyndon's $\mathbf{L}$ which fail to be inherently nonfinitely based. The algebra $\mathbf{A}$ was constructed as the quotient algebra of a subalgebra of $\mathbf{R}^{\mathbb{Z}}$ which in turn was mostly comprised of orbits of the two $\mathbb{Z}$-tuples $\mathbf{a}_{0}$ and $\mathbf{a}_{1}$. We abstract from this construction the following notions.

A basic translation of an algebra $\mathbf{A}$ is a function from $A$ into $A$ obtained from some fundamental operation of $\mathbf{A}$ by assigning values from $A$ to all but one of the places in the operation. By a sink set of an algebra $\mathbf{A}$ we mean a subset of $A$ closed with respect to all basic translations. If $H$ is a sink set of $\mathbf{A}$, then the equivalence relation whose blocks are $H$ and all the singletons $\{a\}$ for $a \in A \backslash H$ will be a congruence. Observe that if $Y$ is a sink set for the algebra $\mathbf{Q}$ and $H$ is the set of all tuples in $Q^{X}$ in which some element of $Y$ occurs, then $H$ is a sink set of $\mathbf{Q}^{X}$. Once a sink set has been designated, we will refer to its elements as sinking elements.

Suppose that $\mathbf{A}$ is an algebra with an automorphism $\sigma$ and a sink set $H$. Let $a_{0}, \ldots, a_{n-1}$ be elements of $A \backslash H$ from distinct $\sigma$-orbits. Let $B$ be the union of the $\sigma$-orbits of the $a_{i}$ 's. A binary operation - is convex on $B$ provided that whenever $\ell, m<n$ and $i$ and $j$ are integers it must be that either $\sigma^{i}\left(a_{\ell}\right) \cdot \sigma^{j}\left(a_{m}\right) \in H$ or else $\sigma^{i}\left(a_{\ell}\right) \cdot \sigma^{j}\left(a_{m}\right)=\sigma^{k}\left(a_{p}\right)$ for some $p<n$ and some $k$ between $i$ and $j$. The magnitude of a binary operation - on the set $B$ is the smallest natural number $d$ such that $\sigma^{i}\left(a_{\ell}\right) \cdot \sigma^{j}\left(a_{m}\right) \notin H$ entails that $|i-j|<d$. An operation need not have a finite magnitude. The notions of convexity and magnitude extend easily to operations of higher ranks.

Theorem 9. Let $\mathbf{Q}$ be a finite groupoid with an absorbing element and with a designated sink set. Let $\mathbf{a}$ and $\mathbf{b}$ be two $\mathbb{Z}$-tuples of nonsinking elements of $Q$. If

(a.) the fundamental operation of $\mathbf{Q}^{\mathbb{Z}}$ is convex on the set consisting of all the shifts of $\mathbf{a}$ and all the shifts of $\mathbf{b}$,

(b.) the fundamental operation of $\mathbf{Q}^{\mathbb{Z}}$ is of finite magnitude on the set consisting of all the shifts of $\mathbf{a}$ and $\mathbf{b}$,

(c.) $\sigma(\mathbf{a})=\sigma(\mathbf{b}) \mathbf{a}$ in $\mathbf{Q}^{\mathbb{Z}}$, where $\sigma$ is the shift automorphism, and

(d.) the equation $\epsilon_{n}$ holds in $\mathbf{Q}$ for all large enough natural numbers n,

Then $\beta_{\mathbf{Q}}$ dominates a strictly increasing linear function. 
Proof. This proof, in essence, repeats the reasoning applied to McKenzie's automatic algebra $\mathbf{R}$. Here the set $A^{\prime}$ will consist of all the shifts of $\mathbf{a}$ and $\mathbf{b}$ as well as all $\mathbb{Z}$-tuples which have sinking entries. The blocks of the congruence $\theta$ are once more the singletons of the shifts of the $\mathbf{a}$ and of $\mathbf{b}$, with one big block containing the tuples with sinking entries. That $\theta$ is actually a congruence follows from the definition of sinking element. The algebra $\mathbf{A}:=\mathbf{A}^{\prime} / \theta$ and the shift automorphism $\sigma$ have all the properties needed the carry out the construction of $\mathbf{G}_{n, 3}$ in the proof of Theorem 1. The magnitude of the operation determines the value of the parameter $d$, while the stipulation that the operation is convex ensures that $w_{L}$ and $w_{R}$ are no greater than 1 . The parameter $\ell$ in that construction can be chosen as a linear function of $n$. This entails that the cardinality of $\mathbf{G}_{n, 3}$ will also depend linearly on $n$. The failure of $\epsilon_{\ell}$ in $\mathbf{G}_{n, 3}$ proceeds exactly as in the reasoning for $\mathbf{R}$, in view of condition (c) above. Our conclusion now follows as in the example with $\mathbf{R}$.

Theorem 9 is easy to apply to automatic algebras. In the example of McKenzie's automatic algebra $\mathbf{R}$ the two $\mathbb{Z}$-tuples

$$
\begin{aligned}
& (\ldots, q, q, q, r, r, r, \ldots) \\
& (\ldots, c, c, c, a, b, b, b, \ldots)
\end{aligned}
$$

represent a single tour through the finite automata - the first tuple records the vertices or states encountered on the tour, while the second records the labels of the edge transited. Condition (c) of Theorem 9 is a consequence of this fact. So we arrive at the following corollary.

Corollary 10. Let $\mathbf{A}$ be a finite automatic algebra. Let $\mathbf{a}$ be a $\mathbb{Z}$-tuple of states and let $\mathbf{b}$ be a $\mathbb{Z}$-tuple of letters which together represent a tour through the underlying finite automata. If

(a.) the fundamental operation of $\mathbf{A}^{\mathbb{Z}}$ is convex on the set consisting of all the shifts of $\mathbf{a}$ and all the shifts of $\mathbf{b}$,

(b.) the fundamental operation of $\mathbf{A}^{\mathbb{Z}}$ is of finite magnitude on the set consisting of all the shifts of $\mathbf{a}$ and $\mathbf{b}$, and

(c.) the equation $\epsilon_{n}$ holds in $\mathbf{A}$ for all large enough natural numbers $n$,

then $\beta_{\mathbf{A}}$ dominates a strictly increasing linear function.

A variation of Theorem 9 has easy application to graph algebras. For this purpose, we need another list of test equations:

$$
x_{0} x_{n-1} x_{n-2} \ldots x_{1} x_{0} x_{n-1} x_{n-2} \ldots x_{1} x_{0} \approx x_{0} x_{n-1} x_{n-2} \ldots x_{1} x_{0}, \quad\left(\delta_{n}\right)
$$

where the product is associated to the right.

Theorem 11. Let $\mathbf{Q}$ be a finite groupoid with an absorbing element and with a designated sink set. Let $\mathbf{a}$ be a $\mathbb{Z}$-tuple of nonsinking elements of $Q$. If 
(a.) the fundamental operation of $\mathbf{Q}^{\mathbb{Z}}$ is convex on the set consisting of all the shifts of $\mathbf{a}$,

(b.) the fundamental operation of $\mathbf{Q}^{\mathbb{Z}}$ is of finite magnitude on the set consisting of all the shifts of $\mathbf{a}$,

(c.) $\sigma(\mathbf{a})=\sigma(\mathbf{a}) \mathbf{a}$ in $\mathbf{Q}^{\mathbb{Z}}$, where $\sigma$ is the shift automorphism, and

(d.) the equation $\delta_{n}$ holds in $\mathbf{Q}$ for all large enough natural numbers n,

Then $\beta_{\mathbf{Q}}$ dominates a strictly increasing linear function.

The proof of Theorem 11 is a bit simpler than the proof sketched for Theorem 9 since the shift automorphism has only one proper orbit rather than two. The demonstration that $\delta_{\ell}$ fails in $\mathbf{G}_{n, 3}$ in this case, requires only a small adjustment of the demonstration that $\epsilon_{\ell}$ fails in the previous case. We omit the details but provide an application. Other applications can be found in Section 5 .

Murskiǐ's graph algebra $\mathbf{A}_{\mathbf{M}}$ was displayed in Figure 1. The graph $\mathbf{M}$ has two vertices $g$ and $h$ connected by an edge and there is a loop at $h$. Let the $\mathbb{Z}$-tuple

$$
\mathbf{a}=(\ldots h, h, h, g, h, g, h, h, g, h, h, h, g, h, h, h, h, g, \ldots) .
$$

Then the operation is convex on the shifts of $\mathbf{a}$ and has magnitude 1 . The verification of $\delta_{n}$ in $\mathbf{A}_{\mathbf{M}}$ is routine. Therefore, $\beta_{\mathbf{A}_{\mathbf{M}}}$ dominates a strictly increasing linear function.

Observe that the $\mathbb{Z}$-tuple $\mathbf{a}$ is a tour through the graph $\mathbf{M}$. Condition (c) of Theorem 11 is a consequence of this fact. So we have the following corollary.

Corollary 12. Let $\mathbf{G}$ be a finite graph. Let $\mathbf{a}$ be a $\mathbb{Z}$-tuple of vertices of $\mathbf{G}$ which is a tour through $\mathbf{G}$. If

(a.) the fundamental operation of $\mathbf{A}_{\mathbf{G}}^{\mathbb{Z}}$ is convex on the set consisting of all the shifts of $\mathbf{a}$,

(b.) the fundamental operation of $\mathbf{A}_{\mathbf{G}}^{\mathbb{Z}}$ is of finite magnitude on the set consisting of all the shifts of $\mathbf{a}$, and

(c.) the equation $\delta_{n}$ holds in $\mathbf{A}_{\mathbf{G}}$ for all large enough natural numbers n,

then $\beta_{\mathbf{A}_{\mathbf{G}}}$ dominates a strictly increasing linear function.

\section{Upper Bounds on Varieties and Algebras}

Let $\mathcal{V}$ be a variety of finite signature and let $\mathbf{B}$ be an algebra of the same signature with fewer than $n$ elements. If $\mathbf{B} \notin \mathcal{V}$ then there must be an equation (of least length) true in $\mathcal{V}$ which fails in $\mathbf{B}$ and, because $\mathbf{B}$ has at most $n-1$ elements, this equation can be chosen so that the only variables occurring in it are among $x_{0}, x_{1}, \ldots, x_{n-2}$. So this equation witnesses that $\mathbf{B} \notin \mathcal{V}^{(n-1)}$. Birkhoff [6] proved that if $\mathcal{V}$ is a locally finite variety of finite signature, then $\mathcal{V}^{(m)}$ is finitely based for every natural number $m$. The proof provides a way to an upper bound on $\beta_{\mathcal{V}}$. Here are the steps in Birkhoff's proof. 
Step I. Since $\mathcal{V}$ is locally finite, the algebra $\mathcal{V}$-freely generated by $\left\{x_{0}, x_{1}, \ldots, x_{n-2}\right\}$ is finite and it elements are classes of terms on $\left\{x_{0}, x_{1}, \ldots, x_{n-2}\right\}$ equivalent modulo the equational theory of $\mathcal{V}$.

Step II. From each equivalence class pick a representative term of shortest length. Step III. Form the finite basis for $\mathcal{V}^{(n-1)}$ by gathering all equations of the form

$$
Q t_{0} t_{1} \ldots t_{r-1} \approx s
$$

where $Q$ is any basic operation symbol, $r$ is the rank of $Q, t_{0}, t_{1}, \ldots, t_{r-1}$ are any representative terms, and $s$ is the term representing the equivalence class to which $Q t_{0} t_{1} \ldots t_{r-1}$ belongs.

There may be several ways to pick the representative terms, so several different finite bases of $\mathbf{V}^{(n-1)}$ may arise in this way. Of course, $\mathcal{V}^{(n-1)}$ can also have other and even simpler bases. In any case, let $\pi_{\mathcal{V}}(n-1)=(r+1) \ell+1$ where $r$ is the largest of the ranks of the finitely many operation symbols and $\ell$ is the length of the longest representative term. Then $\pi_{\mathcal{V}}(n-1)$ is an upper bound on the length of any of the equations obtained by the method of Birkhoff described above. The function $\pi_{\mathcal{V}}$ is called the Birkhoff bound of $\mathcal{V}$. Observe that if $\mathcal{W}$ is a subvariety of $\mathcal{V}$, then $\pi_{\mathcal{W}}(m) \leq \pi_{\mathcal{V}}(m)$ for all $m$. We use $\pi_{\mathbf{A}}$ to stand for $\pi_{\mathcal{V}}$ where $\mathcal{V}$ is the variety generated by the algebra $\mathbf{A}$.

Evidently, $\beta_{\mathcal{V}}(n) \leq \pi_{\mathcal{V}}(n-1)$ for all $n$ and for all locally finite varieties $\mathcal{V}$ of finite signature. Tighter upper bounds are not excluded. Determining the Birkhoff bound is closely related to finding normal forms for terms.

\section{Applications to Graph Algebras and Automatic Algebras}

\subsection{Graph Algebras}

In her seminal dissertation [35], Caroline Shallon associated to each term of the signature of graph algebras a certain rooted graph. A rooted graph is a graph with a designated vertex called the root of the graph. Let $t$ be some term. The term graph $H_{t}$ of $t$ is a rooted graph whose vertex set is the set of variables occurring in $t$ where the leftmost variable of $t$ is the root, and where two vertices $x$ and $y$, perhaps the same, are adjacent if and only if $t$ has a subterm $r \cdot s$ where $x$ and $y$, in some order, are the leftmost variables of $r$ and $s$. The graph $H_{t}$ is always connected. We understand $H_{t}$ as a rooted graph with labeled vertices.

We recall here some results from [35] about the graph algebra belonging to the graph $\mathbf{S}$ displayed in Figure 4.

Theorem 13 (Shallon 1979). Every graph algebra belongs to the variety generated by $\mathbf{A}_{\mathbf{S}}$. An equation $s \approx t$ is true in $\mathbf{A}_{\mathbf{S}}$ if and only if $H_{s}=H_{t}$.

Accordingly, the Birkhoff bound $\pi_{\mathbf{A}_{\mathbf{S}}}(n-1)$ is an upper bound on the equational complexity $\beta_{\mathbf{A}_{\mathbf{G}}}(n)$ for any graph $\mathbf{G}$. Moreover, using the theorem above we can calculate the Birkhoff bound of $\mathbf{A}_{\mathbf{S}}$. Since Shallon's 1979 dissertation is not widely available, we include here a sketch of her proof. 


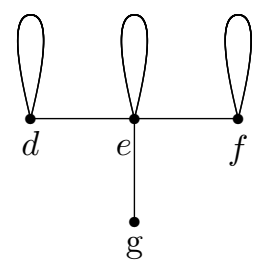

Fig. 4. The Shallon Graph $\mathbf{S}$

Proof Sketch for Theorem 13. Any evaluation of a term $t$ in a graph algebra will result either in the default element 0 or in the value assigned to the root of $H_{t}$. In the event that 0 is not the result of such an evaluation, then the edges of $H_{t}$ provide a record of the products which were computed in the course of the evaluation and the assignment of graph vertices to the variables of $t$ underlying the evaluation associates edges of $H_{t}$ with edges in the graph of the graph algebra. In the event that 0 is the result of the evaluation, then either 0 is assigned to a variable of $t$ or else one of the edges in $H_{t}$ is associated to vertices in the graph of the graph algebra which are not joined by an edge.

Let $s$ and $t$ be terms so that $H_{s}=H_{t}$ and let $\mathbf{G}$ be a graph. We contend that $s \approx t$ is true in $\mathbf{A}_{\mathbf{G}}$. Observe that the same variables occur in $s$ and $t$, so any assignment of 0 to one of these variables will result in assigning $s$ and $t$ the same value, namely 0 . So consider any assignment of vertices of $\mathbf{G}$ to the variables. The value assigned to $t$ is the value assigned to the root of $H_{t}$ unless an edge of $H_{t}$ is associated to vertices of $\mathbf{G}$ which are not joined by an edge. The same applies to $s$. Since $H_{s}=H_{t}$, our assignment must give the same value to $s$ and $t$. This means $s \approx t$ is true in $\mathbf{A}_{\mathbf{G}}$ as we contended.

On the other hand, let $s$ and $t$ be terms so that $H_{s} \neq H_{t}$. We contend that $s \approx t$ fails in $\mathbf{A}_{\mathbf{S}}$. There are four cases depending on why $H_{s} \neq H_{t}$. First, $s$ and $t$ might have different variables. Say $x$ occurs in $s$ but not in $t$. Assign 0 to $x$ and $e$ so all the other variables. Then $s$ is assigned the value 0 while $t$ is assigned the value $e$, and $s \approx t$ fails in $\mathbf{A}_{\mathbf{S}}$. So from here on we suppose that the same variables occur in $s$ and $t$. Second, suppose $H_{s}$ and $H_{t}$ have different roots. Assign $d$ to the root of $H_{s}, e$ to all the other variables. Under this assignment $s$ is given the value $d$ and $t$ is given the value $e$, so once again $s \approx t$ fails in $\mathbf{A}_{\mathbf{S}}$. Third, suppose there is a variable $x$ so that $x$ is joined to itself by an edge in $H_{s}$ but not in $H_{t}$. Assigning $g$ to $x$ and $e$ to all the other variables results in assigning 0 to $s$ and $e$ or $g$ to $t$. Fourth and last, suppose there are distinct variables $x$ and $y$ joined by an edge in $H_{s}$ but not in $H_{t}$. Assign $d$ to $x$ and $f$ to $y$ with $e$ assigned to all the other variables. Under this assignment $s$ is given the value 0 while $t$ is given $d, e$, or $f$ depending on which variable is assigned to the root. So our second contention holds.

The theorem follows from the two contentions we just verified.

Lemma 14. The Birkhoff bound of $\mathbf{A}_{\mathbf{S}}$ is $\pi_{\mathbf{A}_{\mathbf{S}}}(n)=3 n^{2}+3 n+4$. 
Proof. In order to find the Birkhoff bound, we need only determine the length of the appropriate representative terms. So consider a term $t$ whose variables are $x_{0}, \ldots, x_{n-1}$. Now every term equivalent to $t$ must have the same term graph $H_{t}$. Each edge in the term graph corresponds to at least one occurrence of an operation in any associated term. So if there are $k$ edges in $H_{t}$ and $H_{t}=H_{s}$, then $s$ must have length at least $2 k+1$. Emil Kiss [19] gave a method for constructing a term $s$ from $H_{t}$ so that $H_{t}=H_{s}$ and the length of $s$ is this minimum $2 k+1$. Thus, the longest representative term we need is the one associated with the complete graph (with loops at each vertex) on $n$ vertices. This graph has $\left(\begin{array}{l}n \\ 2\end{array}\right)+n$ edges. So it representative term has length $2\left(\left(\begin{array}{l}n \\ 2\end{array}\right)+n\right)+1=n^{2}+n+1$. Therefore $\pi_{\mathbf{A}_{\mathbf{S}}}(n)=$ $3\left(n^{2}+n+1\right)+1=3 n^{2}+3 n+4$, as desired.

Theorem 15. Let $\mathbf{G}$ be a finite graph. Either $\beta_{\mathbf{A}_{\mathbf{G}}}(n)$ is dominated by a constant or $\beta_{\mathbf{A}_{\mathbf{G}}}(n)$ dominates a strictly increasing linear function and, in turn, it is dominated by $3 n^{2}-3 n+4$.

Proof. Baker, McNulty, and Werner [3] showed that $\mathbf{A}_{\mathbf{G}}$ is finitely based if and only if none of the graphs depicted in Figure 5 is an induced subgraph of $\mathbf{G}$. Baker, McNulty, and Werner [2] also showed that the graph algebras associated with the four graphs in Figure 5 are inherently nonfinitely based by showing they fulfill condition (a) of Corollary 12. On the other hand, Theorem 13 entails that every graph algebra satisfies the equation $\delta_{n}$ for every $n$. It follows from Corollary 12 that the equational complexity of such graph algebras dominates some strictly increasing linear function. On the other hand, $3 n^{2}-3 n+4=3(n-1)^{2}+3(n-1)+4$ is an upper bound by Lemma 14 .

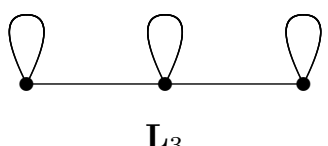

$\mathbf{L}_{3}$

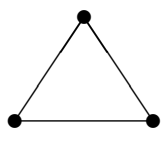

$\mathbf{T}$

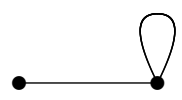

M

Fig. 5. The Four Forbidden Subgraphs 


\subsection{Automatic Algebras}

The theory of automatic algebras is less developed than the theory of graph algebras. In particular, we do not have an analog of Shallon's graph algebra $\mathbf{S}$ nor of Theorem 13. Instead, we consider seven particular examples: McKenzie's algebra $\mathbf{R}$, Lyndon's algebra $\mathbf{L}$, and four variants $\mathbf{L}^{\star}, \mathbf{L}^{\prime}, \mathbf{L}^{\prime \prime}, \mathbf{L}^{\prime \prime \prime}$ of Lyndon's algebra, and the algebra $\mathbf{L}_{3}^{*}$, which is related to the graph $\mathbf{L}_{3}$ and was shown inherently nonfinitely based by Kearnes and Willard in [17]. In 2008, Edmond W. H. Lee [22] proved that $\mathbf{L}^{\star}$ generates the same variety as Lyndon's algebra $\mathbf{L}$. We also consider the four element algebra $\mathbf{V}$ of Višin [38], which is almost an automatic algebra. Except for Lyndon's algebra $\mathbf{L}$ and for the algebra $\mathbf{L}^{\star}$, these algebras are inherently nonfinitely based via the shift automorphism method and their equational complexities dominate strictly increasing linear functions by way of Corollary 10. Here we will demonstrate that these complexities are also dominated by linear functions, except for $\mathbf{L}_{3}^{*}$ which we prove is dominated by a quadratic function. For easy reference these eight algebras are displayed in Figure 6.

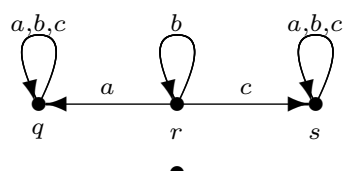

$\mathbf{L}$

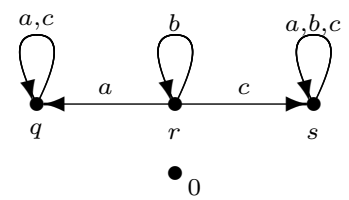

$\mathbf{L}^{\prime \prime \prime}$

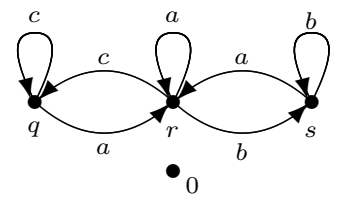

$\mathbf{L}_{3}^{*}$

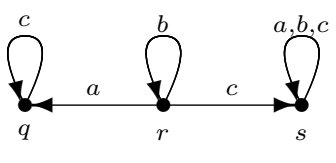

$\bullet{ }_{0}$

$\mathbf{L}^{\prime}$

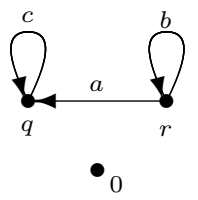

$\mathbf{R}$

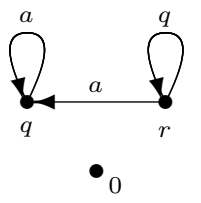

V

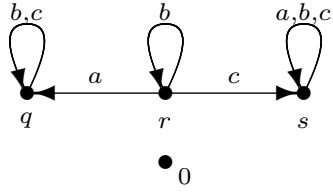

$\mathbf{L}^{\prime \prime}$

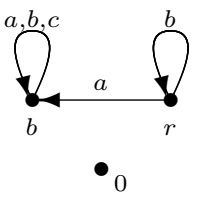

$\mathbf{L}^{\star}$

Fig. 6. Seven automatic algebras and Višin's algebra

Višin's algebra $\mathbf{V}$ fails to be an automatic algebra only because we stipulated that for the underlying automaton the alphabet and the state set must be disjoint. 
Actually, the algebra $\mathbf{V}$ is dually isomorphic (in the sense of interchanging the order of inputs to the basic operation) to the algebra given by Višin in [38]. The variants $\mathbf{L}^{\prime}, \mathbf{L}^{\prime \prime}, \mathbf{L}^{\prime \prime \prime}$ of Lyndon's algebra differ in which letters have been omitted as labels.

Let $\mathbf{A}$ be one of the automatic algebras displayed in Figure 6. In $\mathbf{A}$, some terms denote constantly 0 functions. This will apply to any term that is not associated to the right and to any term in which the rightmost variable occurs more than once. Terms with either of those properties are called zero terms. Since A has a loop, it follows that the only terms which denote the constantly 0 function are the zero terms. If $s \approx t$ is true in $\mathbf{A}$, then either $s$ and $t$ are both zero terms or they are both nonzero terms. In the latter case, the variables that occur in $s$ occur also in $t$ and vice versa. Also $s$ and $t$ have the same rightmost variable.

Since every nonzero term is associated to the right it is possible to drop the parentheses and the operation symbols and treat such a term as a string of variables. Given a nonzero term $t$ we denote by $t^{*}$ the term obtained from $t$ by retaining both the rightmost and the leftmost occurrence of each variable, but deleting all other occurrences of variables. Here is an example:

$$
\begin{aligned}
t= & x(y(x(z(x(y(x w)))))) \\
& x y x z x y x w \\
& x y z \text { yxw } \\
& x y z y x w \\
t^{*}= & x(y(z(y(x w))))
\end{aligned}
$$

Lemma 16. Let $\mathbf{A}$ be any one of the automatic algebras $\mathbf{R}, \mathbf{L}, \mathbf{L}^{\prime}, \mathbf{L}^{\prime \prime}$, or $\mathbf{L}^{\prime \prime \prime}$ and let $t$ be any nonzero term. Then $t \approx t^{*}$ is true in $\mathbf{A}$.

Proof. While we deal with each of the five algebras in turn, the general line of reasoning is the same in each case.

Let $\mathbf{A}=\mathbf{R}$. Assign elements of $R$ to the variables of $t$. There are just three possibilities for the value given to $t$ under this assignment: $r, q$, and 0 . If the value of $t$ turns out to be $r$ then $r$ must have been assigned to the rightmost variable and all other variables must have been assigned $a$. Under this assignment $t^{*}$ is given the value $r$, the same value that was given to $t$. If the value of $t$ turns out to be $q$, then the rightmost variable must have been assigned either $q$ or $r$. In the first case, all other variables must have been assigned $c$ and this means that $t^{*}$ would get $q$ as well. In the second case, when $r$ is assigned to the rightmost variable, then some variable, say $x$, must have been assigned the value $b$. Evidently, $x$ occurs only once in $t$. All the variables (except the rightmost) to the right of $x$ must have been assigned $a$ and all the variables to the left of $x$ must have been assigned $c$. Thus, under this assignment $t^{*}$ will also get the value $q$. So we see that if $t$ gets either the value $r$ or $q$, then $t^{*}$ will get the same value. Likewise, if $t^{*}$ gets either the value $r$ or $q$, then $t$ will get the same value. The only remaining possibility is that both $t$ 
and $t^{*}$ get the value 0 . So in every case $t$ and $t^{*}$ get the same value. This means $t \approx t^{*}$ is true in $\mathbf{A}$.

Let $\mathbf{A}=\mathbf{L}$. Assign elements of $L$ to the variables of $t$. There are now four possibilities for the value given to $t$ under this assignment: $r, q, s$, and 0 . If the value of $t$ turns out to be $r$, then the rightmost variable must have been assigned $r$ and all the other variables must have been assigned $a$. Consequently, $t^{*}$ gets the value $r$ as well. If the value of $t$ turns out to be $q$, then the rightmost variable must have been assigned either $q$ or $r$. In the first case, the remaining variables must have been assigned values from $\{a, b, c\}$. Under such an assignment, $t^{*}$ will also get the value $q$. In the second case, when $r$ is assigned to the rightmost variable, then some variable, say $x$, is assigned the value $b$ with all variables (except the rightmost) to the right of the rightmost occurrence of $x$ assigned the value $a$, and all variables to the left of the rightmost occurrence of $x$ assigned values from $\{a, b, c\}$. Under such an assignment, $t^{*}$ will get the value $q$. By a similar argument, when $t$ gets the value $s$, then $t^{*}$ also gets the value $s$. So we see that inf $t$ gets one of the values $q, r$, or $s$, then $t^{*}$ must get the same value. Likewise, if $t^{*}$ gets one of the values $q, r$, or $s$, then $t$ gets the same value. The only remaining possibility is that $t$ and $t^{*}$ both get the value 0 . So in every case $t$ and $t^{*}$ get the same value. This means $t \approx t^{*}$ is true in $\mathbf{A}$.

The remaining cases are like the case of $\mathbf{A}=\mathbf{L}$ except when $t$ (or $t^{*}$ ) is assigned $q$ and the rightmost variable is assigned $r$. In the event that $\mathbf{A}=\mathbf{L}^{\prime}$, the reasoning needed is the same that was used when $\mathbf{A}=\mathbf{R}$. Consider the case when $\mathbf{A}=\mathbf{L}^{\prime \prime}$. Then some variable, say $x$, is assigned the value $a$, the variable $x$ occurs once in $t$, all variables (except the rightmost) to the right of $x$ are assigned the value $b$ and all other variables are assigned values from $\{b, c\}$. Under such an assignment, $t^{*}$ is also given the value $q$. Finally, consider the case when $\mathbf{A}=\mathbf{L}^{\prime \prime \prime}$. Then some variable, say $x$, is assigned the value $a$, all variables (except the rightmost) to the right of the rightmost occurrence of $x$ are assigned the value $b$ and all other variables are assigned values from $\{a, c\}$. Under such an assignment, $t^{*}$ is also given the value $q$. It follows, as in the case of $\mathbf{L}$ that $t \approx t^{*}$ is true in $\mathbf{A}$.

Theorem 17. Let $\mathbf{L}$ be Lyndon's automatic algebra. The equational complexity of $\mathbf{L}$ eventually dominates every constant function and, in turn, it is dominated by $12 n-20$.

Proof. As observed by Robert Cacioppo [8], every finite algebra whose equational complexity is dominated by a constant must be either finitely based or inherently nonfinitely based. Lyndon [23] proved that $\mathbf{L}$ is not finitely based and Bajusz, McNulty, and Szendrei [1] proved that it fails to be inherently nonfinitely based. Therefore the equational complexity of $\mathbf{L}$ must eventually dominate every constant function.

For the upper bound, observe that every zero term can be represented, with respect to the equational theory of $\mathbf{L}$ by the term $x x$ and every nonzero term $t$ 
can be represented by $t^{*}$. If $n$ is the number of distinct variables occurring in $t$, then $t^{*}$ has at most $2 n-1$ occurrences of variables. This entails that $4 n-3$ is an upper bound on the length of $t^{*}$. Consequently, $\pi_{\mathbf{L}}(n) \leq 12 n-8$. But then $\beta_{\mathbf{L}}(n) \leq \pi_{\mathbf{L}}(n-1) \leq 12 n-20$.

Our methods for producing lower bounds on the equational complexity seem only to apply to inherently nonfinitely based algebras. So we have the following

\section{Open Problem}

Does the equational complexity of Lyndon's Algebra $\mathbf{L}$ dominate a strictly increasing linear function?

The same question might be asked of any nonfinitely based finite algebra which fails to be inherently nonfinitely based.

Theorem 18. Let $\mathbf{A}$ be McKenzie's automatic algebra $\mathbf{R}$ or one the variants $\mathbf{L}^{\prime}, \mathbf{L}^{\prime \prime}$, or $\mathbf{L}^{\prime \prime \prime}$ of Lyndon's automatic algebra. The equational complexity of $\mathbf{A}$ dominates some strictly increasing linear function and, in turn, is dominated by $12 n-20$.

Proof. The upper bounds are established by the same reasoning used in the proof of Theorem 17.

For the lower bounds, we already established just before Theorem 9 that

$$
\frac{1}{36} n-\frac{37}{18} \leq \beta_{\mathbf{R}}(n) \text {. }
$$

So we have to deal with the three variants of Lyndon's automatic algebra. We will invoke Theorem 9. First observe that each of the equations $\left(\epsilon_{n}\right)$ holds in each of these algebras. Also notice that $\{0, s\}$ is a sink set for each of these algebras. To use Theorem 9 we have to find appropriate $\mathbb{Z}$-tuples a and $\mathbf{b}$. Actually, the same two tuples work in all three cases.

$$
\begin{aligned}
\mathbf{a} & :=(\ldots, q, q, q, q, r, r, r, \ldots) \\
\mathbf{b} & :=(\ldots, c, c, c, a, b, b, b, \ldots)
\end{aligned}
$$

Checking that all the hypotheses of Theorem 9 hold under these choices is routine. Consequently, the equational complexity of each of the algebras $\mathbf{L}^{\prime}, \mathbf{L}^{\prime \prime}$, and $\mathbf{L}^{\prime \prime \prime}$ must dominate some strictly increasing linear function.

Theorem 19. The equational complexity of $\mathbf{L}_{3}^{*}$ dominates some strictly increasing linear function and, in turn is dominated by $4 n^{2}-20 n+32$.

Proof. We invoke Theorem 9 to establish the lower bound. Observe that each of the equations $\left(\epsilon_{n}\right)$ holds in $\mathbf{L}_{3}^{*}$ and that $\{0\}$ is a sink set. Here are the appropriate $\mathbb{Z}$-tuples:

$$
\begin{aligned}
\mathbf{a} & :=(\ldots, q, q, q, r, s, s, s, \ldots) \\
\mathbf{b} & :=(\ldots, c, c, c, a, b, b, b, \ldots) .
\end{aligned}
$$


So according to Theorem 9 the equational complexity of $\mathbf{L}_{3}^{*}$ dominates a strictly increasing linear function.

To obtain the upper bound, it is useful to observe that the equations

$$
(x y) z \approx w w \quad x(y y) \approx z z \quad x(x y) \approx x y
$$

are true in $\mathbf{L}_{3}^{*}$. It is harmless to suppose that they belong to any Birkhoff base. The representative terms must all be right-associated:

$$
x_{0}\left(x_{1} \ldots\left(x_{\ell-1} y\right)\right)
$$

and $y \notin\left\{x_{0}, \ldots, x_{\ell-1}\right\}$. Note that the variables $x_{0}, \ldots, x_{\ell-1}$ need not be distinct, but in view of the last equation listed above we can insist that $x_{i} \neq x_{i+1}$ for all $i<\ell-1$. So, in addition to the three equations listed, when forming a base for $\mathcal{V}^{(n-1)}$ we only need equations of the form $x s \approx t$ where $s$ and $t$ are representative terms and $x$ is a variable. The length of such an equation is bounded above by $2 \pi+2$ where $\pi$ is an upper bound on the length of representative terms.

Let $t$ be any term of the form $x_{0}\left(x_{1} \ldots\left(x_{\ell-1} y\right)\right)$ where $y \notin\left\{x_{0}, \ldots, x_{\ell-1}\right\}$ and $x_{i} \neq x_{i+1}$ for all $i<\ell-1$. We contend, as noted without proof by Kearnes and Willard in [17, Lemma 9], that if $s$ is any right associated term on the same variables then $\mathbf{L}_{3}^{*} \models t \approx s$ if and only if $s$ and $t$ have the same leftmost variables, the same rightmost variables, and $H_{t}=H_{s}$. Here $H_{t}$ is the term graph defined at the beginning of Section 5.1. To establish the contention consider any assignment of elements of $\mathbf{L}_{3}^{*}$ to the variables of $s$ (and $t$ ). First suppose that the three conditions hold. Then such an assignment will result in the value 0 for both $s$ and $t$ if 0 is assigned to any variable, or if a letter is assigned to $y$ or if a state is assigned to any of the $x_{i}$ 's, or if $b$ and $c$ are assigned to adjacent variables, or if $x_{\ell-1} y$ evaluates to 0 under the assignment. Under all other assignment the value of $s$ and $t$ is $r$ if $x_{0}$ is assigned $a$, it is $q$ is $x_{0}$ is assigned $c$ and it is $s$ if $x_{0}$ is assigned $b$. So in any event, $\mathbf{L}_{3}^{*} \models s \approx t$. For the converse, suppose that $\mathbf{L}_{3}^{*} \models s \approx t$. Then $s$ and $t$ must have the same rightmost variables, since assigning $r$ to $y$ and $a$ to all the other variable gives $t$ (and hence $s$ ) the value $r$. Next, assigning $c$ to $x_{0}, a$ to all the remaining $x_{i}$ 's and $r$ to $y$ must give $t$ (and hence $s$ ) the value $q$. Consequently, $s$ and $t$ must have the same leftmost variables. Finally, to see that $s$ and $t$ have the same adjacencies (that is $H_{s}=H_{t}$ ), suppose that $u$ and $w$ are adjacent in one of $s$ and $t$, say in $s$. Leaving to the reader the case when one of $u$ and $w$ is $y$, assign $b$ to $u$, assign $c$ to $w$, assign $a$ to all the remaining $x_{i}$ 's and assign $r$ to $y$. Under this assignment $s$ gets the value 0 . Since $t$ must also evaluate to 0 , it follows that $u$ and $w$ must be adjacent in $t$. This establishes our contention.

Now suppose $t$ is of the form $x_{0}\left(x_{1} \ldots\left(x_{\ell-1} y\right)\right)$ where $y \notin\left\{x_{0}, \ldots, x_{\ell-1}\right\}$ and $x_{i} \neq x_{i+1}$ for all $i<\ell-1$ and moreover that $t$ is a term in fewer than $n$ variables. We construct a short representative for $t$ by making a walk through the term graph $H_{t}$. Let $T$ be a spanning tree of $H_{t}$. Observe that $y$ is a leaf of $T$. We begin our walk at $x_{0}$, the leftmost variable of $t$ and end our walk at $y$. During the course of this walk we will traverse each edge of $H_{t}$ at least once, but no edge more than twice. We 
make our short representative $s$ of $t$ in the following way: the rightmost variable of $s$ is $y$. We add variables visited in the walk, one at a time, to the left in the reverse order that we encountered them on our walk. This produces a right-associated term $s$ with rightmost variable $y$, leftmost variable $x_{0}$ and with the same adjacencies as $t$.

Here is how we make the walk. Our walk will extend a depth first transversal of $T$ where the transversal is arranged to end at the leaf $y$. This means that we arrange to walk out to each leaf of $T$, backtracking only as needed, arriving at the particular leaf $y$ at the end. We extend this transversal so that any edge of $H_{t}$ not belonging to $T$ is visited the first time any of its endpoints is hit, with immediate return. Now the leaf $y$ is joined to the rest of $H_{t}$ by only a single edge. This means that, except for the last step, our walk happens on the graph $H_{t}-\{y\}$ which has no more than $n-2$ vertices and, therefore, no more than $\left(\begin{array}{c}n-2 \\ 2\end{array}\right)$ edges. This means the number of times edges are traversed in our walk is no more than $2\left(\begin{array}{c}n-2 \\ 2\end{array}\right)+1=(n-2)(n-3)+1$. Each time an edge is traversed in our walk another operation symbol is added to our representative term $s$. This means the length of $s$ is no more than $\pi=2((n-2)(n-3)+1)+1=2(n-2)(n-3)+3$.

As noted above, the equations needed in the Birkhoff base of $\mathcal{V}^{(n-1)}$ have length bounded by $2 \pi+2=4(n-2)(n-3)+8=4 n^{2}-20 n+32$.

Kearnes and Willard observed, in essence, that every finite graph $\mathbf{G}$ gives rise to an automatic algebra $\mathbf{G}^{*}$ in the same way that $\mathbf{L}_{3}$ gives rise to $\mathbf{L}_{3}^{*}$. Székely proved [36] that the equational complexity of any automatic algebra arising in this way is dominated by $6 n^{2}-24 n+34$.

Theorem 20. The equational complexity of Višin's algebra $\mathbf{V}$ dominates some strictly increasing linear function and, in turn, is dominated by $42 n^{2}+51 n-8$.

Proof. Višin [38] provides an analysis of the equational theory of $\mathbf{V}$ that is detailed enough to give normal forms for all terms that are associated to the right (as well as for many other terms).

Let $\mathcal{V}$ be the variety generated by $\mathbf{V}$. Let $\mathbf{F}$ be the algebra $\mathcal{V}$-freely generated by the countably infinite set $\left\{x, y, z_{0}, z_{1}, \ldots\right\}$ of distinct variables. We regard the elements of $F$ as equivalence classes of terms, where the equivalence relation is just the equational theory of $\mathbf{V}$. Let $t_{n}$ be the term $z_{n}\left(z_{n-1}\left(\ldots\left(z_{0}(x y)\right) \ldots\right)\right)$. According to Višin, the terms $z_{1}, \ldots, t_{0}, t_{1}, \ldots$ and $x x$ all lie in distinct classes. Let $\mathbf{G}$ be the subalgebra of $\mathbf{F}$ generated by the classes of the terms $t_{n}$ and $z_{n}$ where $n$ runs through the natural numbers. Let $\theta$ be the equivalence relation on $G$ which isolates the designated generators and lumps all the remaining elements into a single equivalence class. Višin provides enough information to conclude that $\theta$ is an congruence of $\mathbf{G}$. Let $\mathbf{U}$ be the quotient algebra. This algebra has an absorbing element (the one big block) which we denote by 0 and two infinite sequences of elements $a_{0}, a_{1}, a_{2}, \ldots$ and $b_{1}, b_{2}, b_{3}, \ldots$ that correspond to $t_{0}, t_{1}, t_{2}, \ldots$ and $z_{1}, z_{2}, z_{3}, \ldots$ In $\mathbf{U}$ we have $b_{i+1} a_{i}=a_{i+1}$ with all other products giving the value 0 . An algebra $\mathbf{A}$ can be found 
among the subalgebras of an ultrapower of $\mathbf{U}$ that consists of 0 and two infinite $\mathbb{Z}$-tuples $\ldots, a_{-2}, a_{-1}, a_{0}, a_{1}, \ldots$ and $\ldots, b_{-2}, b_{-1}, b_{0}, b_{1}, b_{2}, \ldots$ Furthermore, in $\mathbf{A}$ we have $b_{i+1} a_{i}=a_{i+1}$ with all other products giving the value 0 . Unlike for $\mathbf{U}$, for A there is an automorphism with these two $\mathbb{Z}$-tuples as orbits. The construction of $\mathbf{G}_{n, 3}$ from the proof of Theorem 1 can be carried out using $\mathbf{A}$. Since the equations $\epsilon_{n}$ hold in $\mathbf{V}$, we can conclude that the equational complexity of $\mathbf{V}$ bounds some strictly increasing linear function.

It remains to determine an upper bound on the length of representative terms on $n$ variables. A term which denotes a constantly 0 term function in $\mathbf{V}$ is called a zero term. The term $x x$ of length 3 represents the zero terms. Višin constructed representatives for the nonzero right associated terms. Such terms on $n$ variables are represented by terms of length no more than $4 n-3$. Observe that $((x y) z) w$ is a zero term but that $((x y) z)$ is not a zero term and it is not right associated.

To obtain our upper bound we need to find normal forms for the nonzero terms which are not right associated. We accomplish this in two steps. Let $t$ be a nonzero term which is not right associated. It must have a subterm of the form $((x s) q)$ where $x$ is a variable and $s$ and $q$ are terms. Among all such subterms there will be a unique longest one which we denote by $t_{r}$. There is a right associated nonzero term $t^{*}(u)$, with rightmost variable $u$, such that $t=t^{*}\left(t_{r}\right)$. Observe that the variable $u$ occurs only once in $t^{*}(u)$. According the Višin [38] there is a nonzero right-associated term $t^{\circ}(u)$ of length no more than $4 n-3$ so that $t^{*}(u) \approx t^{\circ}(u)$ holds in V. Moreover, $u$ is the rightmost variable of $t^{\circ}(u)$ and it occurs only once. It follows that $t \approx t^{\circ}\left(t_{r}\right)$ holds in $\mathbf{V}$ and that the length of $t^{\circ}\left(t_{r}\right)$ is no more than $4 n-4+\ell$, where $\ell$ is the length of $t_{r}$. So in the second step we will look for a short normal form $\hat{t}_{r}$ for $t_{r}$.

The term $t_{r}$ is a nonzero term which denotes a term function $t_{r}^{\mathbf{V}}$ on $\mathbf{V}$ whose only outputs are 0 and $r$. Let $X$ be the set of variables occurring in $t_{r}$. A nontrivial assignment for $t_{r}$ is a function $\alpha: X \rightarrow\{a, r, q\}$ such that $t_{r}^{\mathbf{V}}(\alpha)=r$. We break $X$ into the following pairwise disjoint sets:

$$
\begin{aligned}
A_{t_{r}} & =\left\{x \in X \mid\{a\}=\left\{\alpha(x) \mid \alpha \text { is a nontrivial assignment for } t_{r}\right\}\right\} \\
R_{t_{r}} & =\left\{y \in X \mid\{r\}=\left\{\alpha(y) \mid \alpha \text { is a nontrivial assignment for } t_{r}\right\}\right\} \\
Q_{t_{r}} & =\left\{z \in X \mid\{q\}=\left\{\alpha(z) \mid \alpha \text { is a nontrivial assignment for } t_{r}\right\}\right\} \\
Q A_{t_{r}} & =\left\{w \in X \mid\{q, a\}=\left\{\alpha(w) \mid \alpha \text { is a nontrivial assignment for } t_{r}\right\}\right\} \\
Q R_{t_{r}} & =\left\{u \in X \mid\{q, r\}=\left\{\alpha(u) \mid \alpha \text { is a nontrivial assignment for } t_{r}\right\}\right\} .
\end{aligned}
$$

Because of the structure of $t_{r}$, the sets $A$ and $R$ must be nonempty but the other three sets can turn out to be empty. Pick $x_{0} \in A_{t_{r}}$ and $y_{0} \in R_{t_{r}}$. We make use of the two relations on $Q A$ defined as

$w \bowtie w^{\prime}$ if and only if $\alpha(w)=\alpha\left(w^{\prime}\right)$ for all nontrivial assignments $\alpha$ for $t_{r}$ $w \triangleleft w^{\prime}$ if and only if $\alpha(w)=a$ implies $\alpha\left(w^{\prime}\right)=a$ for all nontrivial assignments $\alpha$ for $t_{r}$.

Observe that $\bowtie$ is an equivalence relation while $\triangleleft$ is a quasiorder which induces a 
partial order on the $\bowtie$ equivalence classes. We need one more relation connecting $Q R_{t_{r}}$ and $Q A_{t_{r}}$. We write $u \prec w$ if and only if $w$ is $\triangleleft$ minimal among all those $x \in Q A$ such that $\alpha(u)=q$ implies $\alpha(x)=a$ for all nontrivial assignments $\alpha$ for $t_{r}$.

The normal form $\hat{t}_{r}$ will also be of the form $((x p) q)$ and it will have its own notion of nontrivial assignment. Our task is to arrange matters so that the two notions of nontrivial assignment turn out to be the same. The five sets of variables and the three binary relations above guide our construction of $\hat{t}_{r}$.

Let $k$ be the maximum of the cardinalities of $A_{t_{r}}$ and $R_{t_{r}}$ and let $j$ be the cardinality of $Q_{t_{r}}$. We take $A_{t_{r}}=\left\{x_{0}, x_{1}, \ldots, x_{k-1}\right\}$ and $R_{t_{r}}=\left\{y_{0}, y_{1}, \ldots, y_{k-1}\right\}$, repeating either $x_{0}$ or $y_{0}$ as needed if $A_{t_{r}}$ and $R_{t_{r}}$ have different cardinalities. We put $Q_{t_{r}}=\left\{z_{0}, z_{1}, \ldots, z_{j-1}\right\}$. We start the construction of $\hat{t}_{r}$ by building one of its subterms $s$

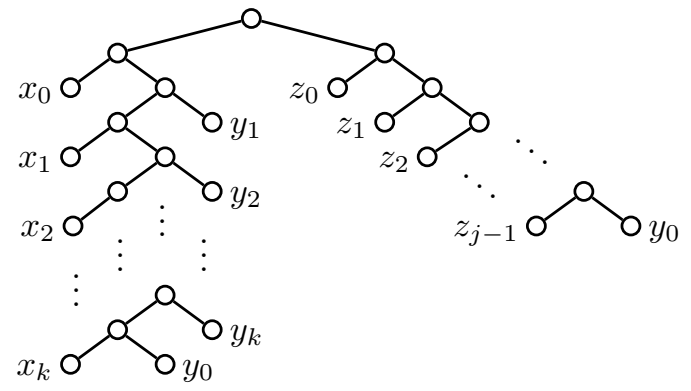

Fig. 7. The term $s$

Let $s$ be the term depicted in Figure 7. It is a nonzero term and it is routine to verify that $A_{t_{r}}=A_{s}, R_{t_{r}}=R_{s}$, and $Q_{t_{r}}=Q_{s}$. The length of $s$ is $2(2 k+j)+1 \leq$ $4 n+1$.

Next we deal with $Q A_{t_{r}}$ and $\bowtie$. Let the equivalence classes with respect to $\bowtie$ be $B_{0}, B_{1}, \ldots, B_{i-1}$. For each equivalence class we create a term. For example, suppose that the elements of $B_{0}$ are $w_{0}, w_{1}, w_{2}$, and $w_{3}$. The corresponding term $b_{0}$ is depicted in Figure 8.

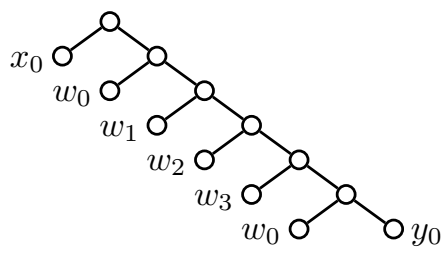

Fig. 8. An example for the term $b_{0}$ 
The length of the term $b_{0}$ is $10=2(4+1)$ where 4 is the size of the equivalence class in our example. Let $m$ be the cardinality of $Q A_{t_{r}}$. The sum of the lengths of the terms $b_{0}, b_{1}, \ldots, b_{i-1}$ is $2(m+i) \leq 4 n$. We put all these terms together with $s$ to form $s^{\prime}$ depicted in Figure 9 .

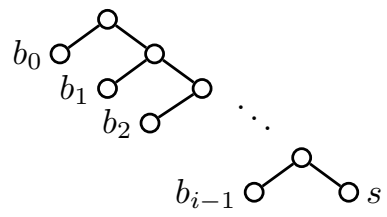

Fig. 9. The term $s^{\prime}$

The term $s^{\prime}$ is again of the same basic form as $t_{r}$ and it is a nonzero term. It is routine to verify that

$$
\begin{aligned}
A_{t_{r}} & =A_{s^{\prime}} \\
R_{t_{r}} & =R_{s^{\prime}} \\
Q_{t_{r}} & =Q_{s^{\prime}} \\
Q A_{t_{r}} & =Q_{s^{\prime}}
\end{aligned}
$$

and that the two versions of $\bowtie$ agree. The length of $s^{\prime}$ is no more than $9 n+1$.

Next we turn to the relation $\triangleleft$. Since this induces a partial order on the $\bowtie$ equivalence classes, we pick representative elements $w_{0} \in B_{0}, w_{1} \in B_{1}, \ldots, w_{i-1} \in$ $B_{i-1}$. For each $\triangleleft$-covering among these representatives we build a term of length 7. For example, if $w_{3}$ covers $w_{5}$, the desired term will be $x_{0}\left(w_{3}\left(w_{5} y_{0}\right)\right)$, which is depicted below.

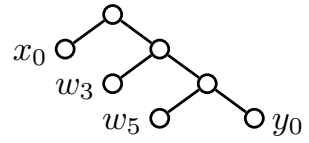

While each such term is only of length 7 , there may be no linear bound on the number of such terms that are needed. On the other hand $n^{2}$ is an upper bound on the number needed. To make the term $s^{\prime \prime}$, we attach all these terms to $s^{\prime}$ in the same manner that we used to build $s^{\prime}$ from $s$. The resulting term $s^{\prime \prime}$ has length no more than $8 n^{2}+9 n+1$. Moreover, the two terms $t_{r}$ and $s^{\prime \prime}$ induce agreement for the sets $A, R, Q$, and $Q A$ and the relations $\bowtie$ and $\triangleleft$.

Last we have to contend with $Q R$ and $\prec$. Suppose that $u \in Q R_{t_{r}}$ and $u \prec w$ where $w$ is one of the representatives selected above. We need the term $d_{w, u}=$ $x_{0}(w u)$ of length 5 . Again, there may be no linear bound on the number of such terms we need, but again we will need no more than $n^{2}$ of them. In case $u \in Q R_{t_{r}}$ but $u \prec w$ fails for all the $w^{\prime} s$, we need the term $d_{u}=x_{0} u$ which has length 3 . 
We need no more than $n$ of these. Finally, we construct $\hat{t}_{r}$ by attaching all these $d$ 's to $s^{\prime \prime}$ in the same manner as before. Thus, the length of $\hat{t}_{r}$ is no more than $14 n^{2}+13 n+1$. It is routine to check that

$$
\begin{aligned}
A_{t_{r}} & =A_{\hat{t}_{r}} \\
R_{t_{r}} & =R_{\hat{t}_{r}} \\
Q_{t_{r}} & =Q_{\hat{t}_{r}} \\
Q A_{t_{r}} & =Q A_{\hat{t}_{r}} \\
Q R_{t_{r}} & =Q R_{\hat{t}_{r}}
\end{aligned}
$$

and in each case the versions of $\bowtie, \triangleleft$, and $\prec$ agree. So we can drop the subscripts.

It remains to show that $\alpha$ is a nontrivial assignment for $t_{r}$ if and only if it is a nontrivial assignment for $\hat{t}_{r}$, for every assignment $\alpha$. Almost by the construction of $\hat{t}_{r}$ we see that if $\alpha$ is nontrivial for $t_{r}$, then it is nontrivial for $\hat{t}_{r}$. So suppose that $\alpha$ is an assignment which is trivial for $t_{r}$. This means that in the process of evaluating $t_{r}$ at $\alpha$ the output 0 is produced at some stage. The argument breaks down into cases according to how the 0 is produced. We call an occurrence of a variable left or right in case it hangs off the tree depicting $t_{r}$ in the given direction. All the variables in $A$ and in $Q A$ occur only in the left sense, while all the variables in $R$ and in $Q R$ occur only in the right sense. It is possible for the variables in $Q$ to have occurrences in both senses.

Case: 0 is assigned by $\alpha$ to some variable. Since the same variables occur in $t_{r}$ and $\hat{t}_{r}$, this means that $\alpha$ is trivial for $\hat{t}_{r}$, as desired.

Now with respect to $\mathbf{V}$ the only products of nonzero elements which produce 0 are $a a, q q, r r, r a, q a$, and $r q$.

Case: One of $a a, r a$, or $q a$ is computed when evaluating $t_{r}$. The only outputs of the operation in $\mathbf{V}$ are $q, r$, and 0 . So in the course of evaluating $t_{r}$ at $\alpha$ neither $a a$ nor $r a$ nor $q a$ arise, unless $a$ is assigned to a variable in $R \cup Q \cup Q R$; in which case $\hat{t}_{r}$ must also evaluate to 0 , as desired.

Case: One of $r r$ or $r q$ is computed when evaluating $t_{r}$. If $\alpha$ assigns $r$ to a variable $x$ occurring to the left in $t_{r}$ then $x \in A \cup Q A \cup Q$. Thus $x$ also occurs to the left in $\hat{t}_{r}$, which entails that $\alpha$ is trivial for $\hat{t}_{r}$. So consider the remaining subcase when one of $(q r) r$ or $(q r) q$ is computed when evaluating $t_{r}$. Recalling that $((x y) z) w$ is a zero term, we see that $\alpha$ assigns $q$ to some variable $x \in A$. This forces $\alpha$ to be trivial for $\hat{t}_{r}$, as desired.

Case: $q q$ is computed when evaluating $t_{r}$. The assignment of $q$ to any variable not in $Q \cup Q R \cup Q A$ will force $\hat{t}_{r}$ to evaluate to 0 . Thus, there are four subcases.

Subcase: $w u$ is a subterm of $t_{r}$ with $w, u \in Q \cup Q A \cup Q R$ and $\alpha(w)=\alpha(u)=q$. If $w \in Q$, then $u \in R$ since $t_{r}$ must have nontrivial assignments. Thus $w \notin Q$. Likewise, if $u \in Q$, then $w \in A$. So $u \notin Q$. Since $w$ occurs to the left in $t_{r}$ and $u$ occurs to the right, we see that $w \in Q A$ and $u \in Q R$. Therefore there must be 
$w^{\prime} \in Q A$ so that $u \prec w^{\prime} \triangleleft w$. But then $\hat{t}_{r}$ has been rigged up in such a way that it must evaluate to 0 at $\alpha$.

Subcase: $(x p) y$ is a subterm of $t_{r}$ and $\alpha(x)=a$ and $\alpha(y)=q$. Evidently, $y \in R$ since $t_{r}$ has nontrivial assignments. Since $\alpha(y)=q$, we conclude that $\hat{t}_{r}$ must evaluate to 0 .

Subcase: $x(y p)$ is a subterm of $t_{r}$ and $\alpha(x)=q$ while $\alpha(y)=a$. The variables $x$ and $y$ occur in $t_{r}$ on the left, so $x, y \in Q \cup Q A \cup A$. If $x \in A$, then $\hat{t}_{r}$ evaluates to 0 since $\alpha(x)=q$. So consider that $x \in Q \cup Q A$. Likewise, if $y \in Q$, then $\hat{t}_{r}$ evaluates to 0 since $\alpha(y)=a$. So consider that $y \in Q A \cup A$. Any assignment $\gamma$ which is nontrivial for $t_{r}$ such that $\gamma(y)=a$ will force $\gamma(x)=a$ as well. Hence, $x \notin Q$. It follows that $x \in Q A$. Pick an assignment $\delta$ which is nontrivial for $t_{r}$ so that $\delta(x)=q$. Then $\delta(y)=q$ as well. It follows that $y \in Q A$ and that $y \triangleleft x$. But this means that $\alpha$ is trivial for $\hat{t}_{r}$ since $\alpha(y)=a$ but $\alpha(x)=q$.

Subcase: $(x p)(y g)$ is a subterms of $t_{r}$ where $\alpha(x)=\alpha(y)=a$. Since $t_{r}$ has nontrivial assignments, so $y \in Q$. But then $\hat{t}_{r}$ evaluates to 0 since $\alpha(y)=a$.

No cases remain since any terms producing $q$ must be of the form $x$ or $x p$, where $x$ is a variable.

Finally, observe that $t \approx t^{*}\left(\hat{t}_{r}\right)$ holds in $\mathbf{V}$ and that the length of $t^{*}\left(\hat{t}_{r}\right)$ is no more than $14 n^{2}+17 n-3$. This entails that $\beta_{\mathbf{V}}(n) \leq 42 n^{2}+51 n-8$, as desired. $\square$

\section{Connections with computational complexity}

In this section we prove the following theorem which uses the equational complexity of $\mathbf{A}$ to obtain an estimate of the time complexity of the finite algebra membership problem for the variety generated by $\mathbf{A}$.

Theorem 21. Let $\mathbf{A}$ be a finite algebra of finite signature. The time complexity of the finite algebra membership problem for the variety generated by $\mathbf{A}$ is eventually dominated by

$$
n^{2 \beta_{\mathbf{A}}(n+1)+n}
$$

where $n$ is the cardinality of the input algebra.

Proof. The method we use to determine whether an algebra $\mathbf{B}$ of cardinality $n$ belongs to the variety generated by $\mathbf{A}$ is simply to check in $\mathbf{B}$ all equations true in $\mathbf{A}$ which have length less than $\beta_{\mathbf{A}}(n+1)$ and in which no more than $n$ distinct variables occur.

We first bound the complexity of checking equations in finite algebras.

Let $\mathbf{B}$ be an algebra of cardinality $n$. We think of this algebra as given by a system of sorted lists, where each list is associated with a basic operation of $\mathbf{B}$. The list associated with a basic operation of rank $r$ is a list of the $r+1$-tuples belonging to the operations. Such a list will have $n^{r}$ entries. 
Let $Q^{\mathbf{B}}$ be a basic operation of $\mathbf{B}$ and let $r$ be its rank. To evaluate $Q^{\mathbf{B}}$ at a given $r$-tuple amounts to searching the system of lists for the correct entry. Using a standard binary search, this requires no more than

$$
c\left(\left\lfloor\log n^{r}\right\rfloor+1\right)
$$

steps, where $c$ is an integral constant that depends on the signature but not on $n$. Since $r$ is also an integer, it follows that $c\left(\left\lfloor\log n^{r}\right\rfloor+1\right) \leq c r(\lfloor\log n\rfloor+1)$. Also notice that $c(\lfloor\log n\rfloor+1) \leq n$ for all large enough $n$. So we see that evaluating $Q^{\mathbf{B}}$ at a given $r$-tuple can be accomplished in no more than $r n$ steps, for all large enough values of $n$.

Evaluation of the term function $s^{\mathbf{B}}$ associated with a term $s$ at a given tuple amounts to systematically evaluating each basic operation as it comes up in the term. To analyze this let $\mathcal{O}$ be the set of operation symbols and let $|s|_{Q}$ stand for the number of occurrences of the operation symbol $Q$ in the term $s$. Then the number of steps needed to evaluate $s^{\mathbf{B}}$ at a given tuple is no more than

$$
\sum_{Q \in \mathcal{O}}|s|_{Q} \operatorname{rank} Q n=n \sum_{Q \in \mathcal{O}}|s|_{Q} \operatorname{rank} Q .
$$

A routine induction on terms shows that $\ell(s)-1=\sum_{Q \in \mathcal{O}}|s|_{Q} \operatorname{rank} Q$, where $\ell(s)$ is the length of $s$. Therefore, the evaluation of $s^{\mathbf{B}}$ at a given tuple can be accomplished in no more than $n(\ell(s)-1)$ steps, for all sufficiently large values of $n$.

Now suppose that $s \approx t$ is an equation in no more than $r$ variables. To check whether this equation is true in $\mathbf{B}$, for each of the $n^{r}$ possible $r$-tuples we need to evaluate $s^{\mathbf{B}}$ and $t^{\mathbf{B}}$ and compare the two values. This can be done in no more than

$$
n(\ell(s)-1)+n(\ell(t)-1)+1=n(\ell(s)+\ell(t)-2)+1 \leq n(\ell(s)+\ell(t))
$$

steps, for each $r$-tuple. Finally, we can check whether $s \approx t$ holds in $\mathbf{B}$ in no more than $[\ell(s)+\ell(t)] n^{r+1}$ steps, provided $n$ is sufficiently large.

Now let $k$ be the cardinality of $\mathbf{A}$ and let $m$ be the number of operation symbols for the signature. The equations of length $\ell$ with variables from $x_{0}, x_{1}, \ldots, x_{r-1}$ are just certain strings of length $\ell$ of symbols drawn from an alphabet with $m+r$ symbols. There are $(m+r)^{\ell}$ such strings, not all of them equations. We consider all $(m+r)^{\ell}$ strings one at a time. For each we test whether it is an equation true in $\mathbf{A}$. This takes no more than $\ell k^{r+1}$ steps. If it turns out to be true, then we test it in $\mathbf{B}$ and this takes no more than $\ell n^{r+1}$ steps. So testing all the strings can be accomplished in no more than

$$
(m+r)^{\ell}\left[\ell k^{r+1}+\ell n^{r+1}\right]=\ell(m+r)^{\ell}\left[k^{r+1}+n^{r+1}\right]
$$

steps.

We are concerned with the case when $r=n$ and $\ell$ ranges from 2 to $\beta_{\mathbf{A}}(n+1)-1$. 
So, assuming $n>m$ and $n>k$ we can bound the number of steps needed by

$$
\begin{aligned}
\left(\beta_{\mathbf{A}}(n+1)\right)^{2}(m+n)^{\beta_{\mathbf{A}}(n+1)-1}\left(k^{n+1}+n^{n+1}\right) & \leq\left(\beta_{\mathbf{A}}(n+1)\right)^{2}(2 n)^{\beta_{\mathbf{A}}(n+1)-1} 2 n^{n+1} \\
& \leq\left(\beta_{\mathbf{A}}(n+1)\right)^{2} 2^{\beta_{\mathbf{A}}(n+1)} n^{\beta_{\mathbf{A}}(n+1)+n} \\
& \leq n^{2 \beta_{\mathbf{A}}(n+1)+n}
\end{aligned}
$$

where the final inequality holds since $b^{2} 2^{b} \leq n^{b}$ when $4<n$ and $b$ is positive.

The 2 that appears in the formula in Theorem 21 can be replaced by any real number larger than 1 . In the event that $\beta_{\mathbf{A}}$ exhibits only polynomial growth, the bound on computational complexity given by this theorem is better than the bound found by Bergman and Slutzki for the brute force algorithm.

\section{References}

[1] Tamás Bajusz, George McNulty, and Ágnes Szendrei, Lyndon's groupoid is not inherently nonfinitely based, Algebra Universalis 27 (1990), no. 2, 254-260. MR1037867 (90m:08007)

[2] Kirby A. Baker, George F. McNulty, and Heinrich Werner, Shift-automorphism methods for inherently nonfinitely based varieties of algebras, Czechoslovak Math. J. 39(114) (1989), no. 1, 53-69. MR983483 (90a:08004)

[3] - The finitely based varieties of graph algebras, Acta Sci. Math. (Szeged) 51 (1987), no. 1-2, 3-15. MR911554 (88m:08007)

[4] Joel Berman, Algebraic properties of k-valued logic, Proceedings of the tenth international symposium on multiple-valued logic (Evanston, Ill., 1980), IEEE, Long Beach, Calif., 1980, pp. 195-204.

[5] Clifford Bergman and Giora Slutzki, Complexity of some problems concerning varieties and quasi-varieties of algebras, SIAM J. Comput. 30 (2000), no. 2, 359-382 (electronic). MR1769362 (2001g:68038)

[6] Garrett Birkhoff, On the structure of abstract algebras, Proc. Camb. Phil. Soc. 31 (1935), $433-454$.

[7] Subdirect unions in universal algebra, Bull. Amer. Math. Soc. 50 (1944), 764-768. MR0010542 (6,33d)

[8] Robert Cacioppo, Non-finitely based pseudovarieties and inherently non-finitely based varieties, Semigroup Forum 47 (1993), no. 2, 223-226. MR1230146 (94g:08008)

[9] Dejan Delić, Finite bases for flat graph algebras, J. Algebra 246 (2001), no. 1, 453-469. MR1872631 (2002j:08007)

[10] Samuel Eilenberg and M. P. Schützenberger, On pseudovarieties, Advances in Math. 19 (1976), no. 3, 413-418. MR0401604 (53 \#5431)

[11] Ralph Freese, George F. McNulty, and J. B. Nation, Inherently nonfinitely based lattices, Ann. Pure Appl. Logic 115 (2002), no. 1-3, 175-193. MR1897025 (2003c:06005)

[12] Ralph Freese, George McNulty, and J. B. Nation, A modular inherently nonfinitely based lattice, Algebra Universalis 55 (2006), no. 2-3, 119-125. Special issue dedicated to Walter Taylor. MR2280221

[13] I. M. Isaev, Inherently non-finitely based varieties of algebras, Sibirsk. Mat. Zh. 30 (1989), no. 6, 75-77 (Russian). MR1043435 (91c:08008)

[14] Marcel Jackson and Ralph McKenzie, Interpreting graph colourability in finite semigroups, Internat. J. Algebra Comp. 16 (2006), no. 1, 119-140. MR2217645 (2006m:20081) 
[15] J. Kalicki, On comparison of finite algebras, Proc. Amer. Math. Soc. 3 (1952), 36-40. MR0047584 (13,898f)

[16] Keith A. Kearnes and Emil W. Kiss, Finite algebras of finite complexity, Discrete Math. 207 (1999), 89-135. MR1710485 (2000k:08001)

[17] Keith A. Kearnes and Ross Willard, Inherently nonfinitely based solvable algebras, Canad. Math. Bull. 37 (1994), no. 4, 514-521. MR1303679 (95i:08009)

[18] Andrei Kelarev, Graph Algebras and Automata, Pure and Applied Mathematics, vol. 257, Marcel Dekker, New York, 2003.

[19] Emil W. Kiss, A note on varieties of graph algebras, Universal algebra and lattice theory (Charleston, S.C., 1984), 1985, pp. 163-166. MR823014 (87c:05108)

[20] Marcin Kozik, On Some Complexity Problems in Finite Algebras, Ph.D. Thesis, Vanderbilt University, 2004.

[21] Gábor Kun and Vera Vértesi, The membership problem in finite flat hypergraph algebras, Internat. J. Algebra Comput. 17 (2007), no. 3, 449-459. MR2333367 (2008f:08008)

[22] Edmond W. H. Lee, Lyndon's groupoid generates a small almost Cross variety, Algebra Universalis (2008), to appear.

[23] R. C. Lyndon, Identities in finite algebras, Proc. Amer. Math. Soc. 5 (1954), 8-9. MR0060482 $(15,676 \mathrm{~b})$

[24] A. I. Mal'cev, Algebraic systems, Springer-Verlag, New York, 1973. MR0349384 (50 \#1878)

[25] Ralph McKenzie, The residual bounds of finite algebras, Internat. J. Algebra Comput. 6 (1996), no. 1, 1-28. MR1371732 (97e:08002a)

[26] - The residual bound of a finite algebra is not computable, Internat. J. Algebra Comput. 6 (1996), no. 1, 29-48. MR1371733 (97e:08002b)

[27] _ Tarski's finite basis problem is undecidable, Internat. J. Algebra Comput. 6 (1996), no. 1, 49-104. MR1371734 (97e:08002c)

[28] George F. McNulty and Caroline R. Shallon, Inherently nonfinitely based finite algebras, Universal algebra and lattice theory (Puebla, 1982), 1983, pp. 206-231. MR716184 (85h:08011)

[29] V. L. Murskiı̌, The existence in the three-valued logic of a closed class with a finite basis having no finite complete system of identities, Dokl. Akad. Nauk SSSR 163 (1965), 815-818 (Russian). MR0186539 (32 \#3998)

[30] - The number of $k$-element algebras with a binary operation which do not have a finite basis of identities, Problemy Kibernet. 35 (1979), 5-27, 208 (Russian). MR539884 (80i:08003)

[31] Jan Reiterman, The Birkhoff theorem for finite algebras, Algebra Universalis 14 (1982), no. 1, 1-10. MR634411 (84c:08008)

[32] M. V. Sapir, Problems of Burnside type and the finite basis property in varieties of semigroups, Izv. Akad. Nauk SSSR Ser. Mat. 51 (1987), no. 2, 319-340, 447 (Russian). MR897000 (88h:20078)

[33] _ Inherently non-finitely based finite semigroups, Mat. Sb. (N.S.) 133(175) (1987), no. 2, 154-166, 270 (Russian). MR905002 (88m:20116)

[34] Mark V. Sapir, Sur la propriété de base finie pour les pseudovariétés de semigroupes finis, C. R. Acad. Sci. Paris Sér. I Math. 306 (1988), no. 20, 795-797 (French, with English summary). MR949035 (89d:08008)

[35] Caroline Shallon, Nonfinitely Based Finite Algebras Derived from Lattices, Ph.D. Thesis, University of California, Los Angeles, 1979.

[36] Zoltán Székely, Complexity of the Finite Algebra Membership Problem for Varieties, Ph.D. Thesis, University of South Carolina, 1998.

[37] _ Computational complexity of the finite algebra membership problem for varieties, Internat. J. Algebra Comput. 12 (2002), no. 6, 811-823. MR1949698 (2003k:08009) 
[38] V. V. Višin, Identity transformations in a four-valued logic, Dokl. Akad. Nauk SSSR 150 (1963), 719-721 (Russian). MR0201292 (34 \#1176)

[39] Brian L. Walter, Finite equational bases for directed graph algebras, Ph.D. Thesis, University of California, Los Angeles, 2002.

[40] _ The finitely based varieties of looped directed graph algebras, Acta Sci. Math. (Szeged) 72 (2006), 421-458. MR2289747 (2007m:08007)

[41] Ross Willard, Tarski's finite basis problem via $A(\mathcal{T})$, Trans. Amer. Math. Soc. 349 (1997), no. 7, 2755-2774. MR1389791 (97i:03019) 